\title{
Investigation of Rotating Stall Phenomenon and Optimization in Mixed-Flow Waterjet Propulsion Device
}

\author{
Can Luo $\left(\mathbb{D},{ }^{1,2}\right.$ Di Zhang, ${ }^{1}$ Congcong Zhang, ${ }^{1}$ Shuaihao Lei, ${ }^{1}$ Chenzhi Xia, ${ }^{3}$ Chuan Wang $\mathbb{D}^{1},{ }^{1}$ \\ and Li Cheng $\mathbb{1}^{1}$ \\ ${ }^{1}$ College of Hydraulic Science and Engineering, Yangzhou University, Yangzhou, Jiangsu Province 225009, China \\ ${ }^{2}$ Key Laboratory of Fluid and Power Machinery of Ministry of Education, Xihua University, Chengdu, Sichuan 610039, China \\ ${ }^{3}$ The Eastern Route of South-to-North Water Diversion Project Jiangsu Water Source Co. Ltd., Nanjing, Jiangsu 210019, China
}

Correspondence should be addressed to Can Luo; luocan@yzu.edu.cn and Li Cheng; chengli@yzu.edu.cn

Received 29 March 2020; Accepted 22 June 2020; Published 23 July 2020

Academic Editor: Alicia E. Ares

Copyright (c) 2020 Can Luo et al. This is an open access article distributed under the Creative Commons Attribution License, which permits unrestricted use, distribution, and reproduction in any medium, provided the original work is properly cited.

The rotating stall is a kind of flow phenomenon in the impeller harming the navigation speed of vessels propelled by a waterjet propulsion device when the waterjet propulsion device operates at the small flow rate conditions. The numerical simulated hydraulic performance was compared with experimental results so that the reliability of the CFD method was verified. The grooves are proposed before the inlet of an impeller to suppress the rotating stall. The orthogonal experiment is designed to seek the appropriate values of the parameters such as the length, width, depth, and number of the groove. The results show that the width of groove has the greatest influence on the performance of the device, followed by the number, the depth, and the length. The width, number, depth, and length of the selected groove scheme are $3.10 \times 10^{-2} \mathrm{D}, 72,3.10 \times 10^{-2} \mathrm{D}$, and $7.75 \times 10^{-2} \mathrm{D}$, respectively. At the rotating stall conditions, the selected groove scheme is numerically calculated. In contradistinction to the original scheme, it is found that the groove can improve the flow in the impeller and enhance the head of valley point condition, but the efficiency drops due to the increasing local hydraulic loss near the groove. When the groove is installed, the positive slop region on the flow ratehead curve disappears related to the static pressure difference on the blade, especially depending on the static pressure on the suction side. The flow pattern on the span surface of the vortex core is smooth, and then, the head increases. On the contrary, the head decreases. The outcome is beneficial to improve the performance of the waterjet propulsion device and enrich the rotating stall theory.

\section{Introduction}

Ship stalls affecting the navigation speed of vessels are divided into wind wave stall and propulsion stall [1]. Wind wave stall refers to the condition that the navigation speed is lower than the calm speed due to the wind and waves when the power of motor is constant [2]. The essence of wind wave stall is the enhancing resistance up to the parameters such as the wave, the length of ship, and the linear square parameters of the hull and the wind. On the contrary, the reason for the propulsion stall is the rotating stall existing in the propellers and waterjet propulsion pumps. The ship applied with the waterjet propulsion device is propelled by using the reaction force of the ejecting water from the nozzle. The core component is the propulsion pump which are the mixedflow and axial flow pump. No matter which kind of pump, rotating stall happens at some conditions probably. $\mathrm{Nu}$ merous research studies have been carried out to analyze the rotating stall by the predecessors [3-6]. Arpad found that the rotating stall is an important reason for the occurrence of unstable hydraulic zone in the axial flow pump [7]. Emmons et al. expounded the mechanism of the occurrence of the rotating stall in detail [8]. The rotating stall generally occurs in the groove channel unstably but can be dynamically transmitted in the neighboring groove channel along the rotating direction. Cavitation in a groove channel causes the increasing attack angle of airfoil of neighboring blade in the rotating direction and then results in enhancing the 
probability of cavitation in the corresponding groove channel of the airfoil. Once the cavitation condition is reached, the cavitation occurs in that groove channel, but cavitation in the groove channel in the opposite direction of rotation tends to disappear. In order to improve the hydraulic conditions in the unstable hydraulic zone and achieve the effect of suppressing the rotating stall, different suppression measures such as separator are adopted $[9,10]$. In recent years, CFD technology matures and is widely applied in fields such as turbo machinery [11-20], valve [21, 22], and other aspects [23-27]. In this study, the numerical simulation analysis of the stall characteristics of the mixed-flow waterjet propulsion device is carried out. The groove installed before the inlet of the impeller is proposed. To seek the key factors affecting the performance of the waterjet propulsion device, the orthogonal test is formulated. The orthogonal method is mature and widely used in many fields such as energy [28], chemistry [29], pharmacy [30], and environment [31]. The hydraulic performance and flow characteristics of the selected and original schemes are compared, and the suppression mechanism is analyzed. This research work not only captures and analyzes the rotating stall phenomenon of the mixed-flow waterjet propulsion device but also puts forward the corresponding solutions, which provides an important reference for other similar devices.

\section{Numerical Simulation Method}

2.1. Governing Equations. The continuity, momentum, and energy equations are adopted in the CFD numerical calculation following the mass conservation, momentum conservation, and energy conservation, called governing equations. Ignoring heat transfer in the waterjet propulsion device, the continuity and momentum equations are kept to describe the flow.

The control body is divided into two types: a finite control body with a fixed space and a finite control body that moves with the fluid. The waterjet propulsion device is regarded as a limited control body in a fixed space. The flow in the control body is a constant viscosity incompressible turbulence. In the Cartesian coordinate system, equations (1) and (2) are the continuity equation and the momentum equation:

$$
\begin{gathered}
\frac{\partial \rho}{\partial t}+\frac{\partial\left(\rho u_{i}\right)}{\partial x_{i}}=0 \\
\frac{\partial\left(\rho u_{i}\right)}{\partial t}+\frac{\partial\left(\rho u_{i} u_{j}\right)}{\partial x_{j}}=-\frac{\partial P}{\partial x_{i}}+\frac{\partial}{\partial x_{j}}\left[\mu\left(\frac{\partial u_{i}}{\partial x_{j}}+\frac{\partial u_{j}}{\partial x_{i}}\right)\right]+F_{i}
\end{gathered}
$$

where $\rho$ is the water density, $u$ is the water speed, $t$ is the time, $x$ is the Cartesian coordinate, $P$ is the pressure acting on the microfluid body, $\mu$ is the dynamic viscosity of water, $i$ and $j$ are the axis direction, and $F$ is the volume force.

For steady-state flow, the density does not change with time, so the changing rate of density is zero, and equation (1) can be written as follows:

$$
\frac{\partial(\rho u)}{\partial x}+\frac{\partial(\rho v)}{\partial y}+\frac{\partial(\rho w)}{\partial z}=0
$$

2.2. Computational Domain and Mesh Generation. Affected by the factors such as the bottom boundary layer of the ship, the flow at the inlet of the waterjet propulsion pump device is uneven. In order to ensure the accuracy of numerical simulation and mesh generation, the complete computational domain is divided into subdomains such as water body, inlet duct, impeller, guide vane, and nozzle, and the inlet duct can be divided into three parts: inlet contraction section, elbow pipe section, and outlet horizontal section, shown in Figure 1. The three-dimensional size of the water body is $30 D, 5 D$, and $8 D$, while $D$ is the diameter of the impeller inlet.

Based on the ANSYS ICEM platform, different mesh strategies are used to complete the structured mesh of each subdomain. The nozzle and the outlet horizontal section of the inlet duct adopt the O-type mesh strategy, and the guide vane and the impeller adopt the H-type mesh strategy. Block structure is established to generate mesh according to the geometric characteristics of inlet contraction section. Considering the complex flow in the impeller, the vane, and near the lips of inlet duct, local intensive mesh is required. When the mesh quantity exceeds 3.35 million, the head and efficiency of the device are basically unchanged. So, meshes of each subdomain in Figure 2 are employed.

2.3. Boundary Conditions. Considering that the inlet of the water at the bottom of the ship is far enough away from the inlet of the device, it is not affected by the boundary layer of the hull, so the inflow is uniform. In order to simulate the ship speed, the inlet is set as the velocity inlet, and the velocity is $8 \mathrm{~m} / \mathrm{s}$; that is, the navigation speed is $8 \mathrm{~m} / \mathrm{s}$. The outlet is set as the average static pressure outlet, and the reference pressure is $1 \mathrm{~atm}$. The wall includes the inlet duct, the shaft, the impeller, the vane, the nozzle, and the other four faces of the water body except the inlet and outlet. The scalable wall function is applied to treat the wall. Since the mesh on both sides of the interface in the neighboring subdomain are not consistent, the GGI grid connection technology is employed to process the interface. Multiple reference frame (MRF) is applied in the coordinate system. The rotating speed of impeller is $700 \mathrm{r} / \mathrm{min}$.

\section{Test Arrangement and Verification}

3.1. Model Test Bench Layout. In order to verify the numerical simulation results, a closed double-cycle waterjet propulsion test system was established, shown in Figure 3. The test consists of a waterjet propulsion device and pipe system, in which the waterjet propulsion device is composed of the impeller, the guide vane, the shaft, and the coupling, and the pipe system is composed of a main circulation system and a secondary circulation system. The main circulation system is used to realize the water circulation of the test bench, providing the navigation speed for the waterjet 


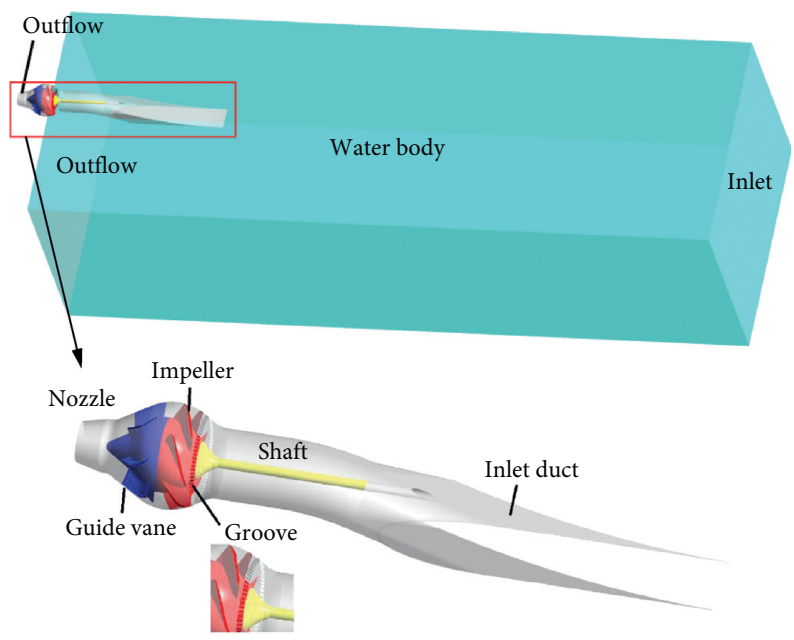

Figure 1: Computational domain.

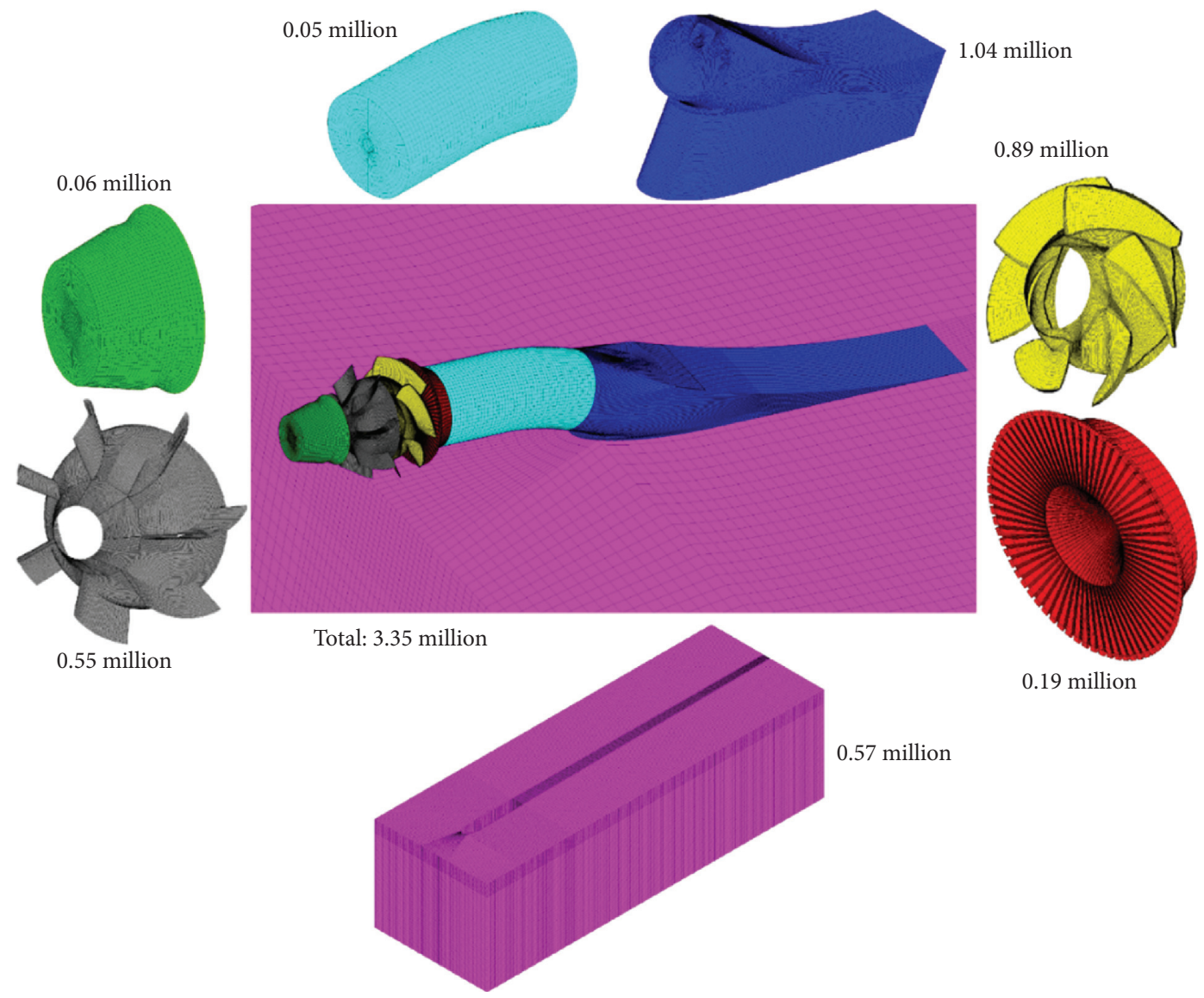

Figure 2: Mesh generation.

propulsion device. The secondary circulation system is the circulation of the waterjet propulsion device. PVC or stainless steel flanges are installed between different parts on the test bench. Electromagnetic flow meters and pressure transmitters are set to measure the flow rate and pressure on the upstream and downstream of the waterjet propulsion device. The function of the butterfly valve is to control the flow rate in the pipe circulation system.
3.2. Test Result Verification. When the rotating speed is $400 \mathrm{rev} / \mathrm{min}$, the hydraulic performance is numerically simulated and tested. Based on the flow rate $Q$, efficiency $\eta$, and head $H$ at the best efficiency point, the dimensionless flow rate $Q^{\prime}$, head $H^{\prime}$, and efficiency $\eta^{\prime}$ are plotted in Figure 4. By comparison, the CFD results and test results are completely consistent, which indicates that the numerical simulation method adopted in this study is feasible and the results are credible. 


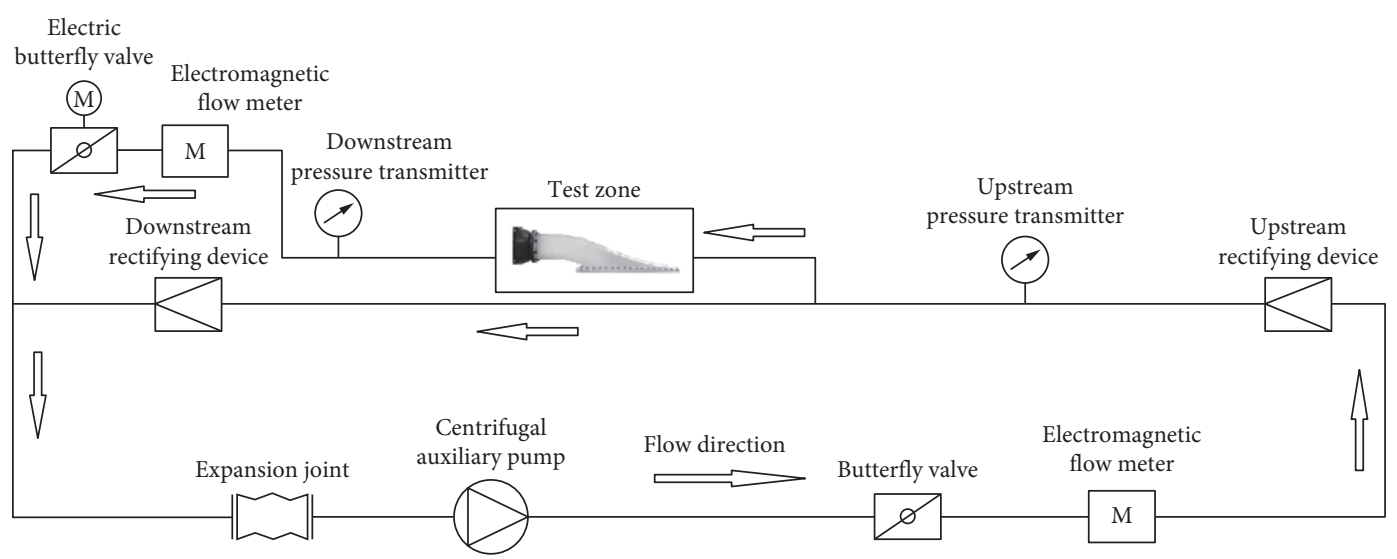

FIGURE 3: Waterjet propulsion device test system.

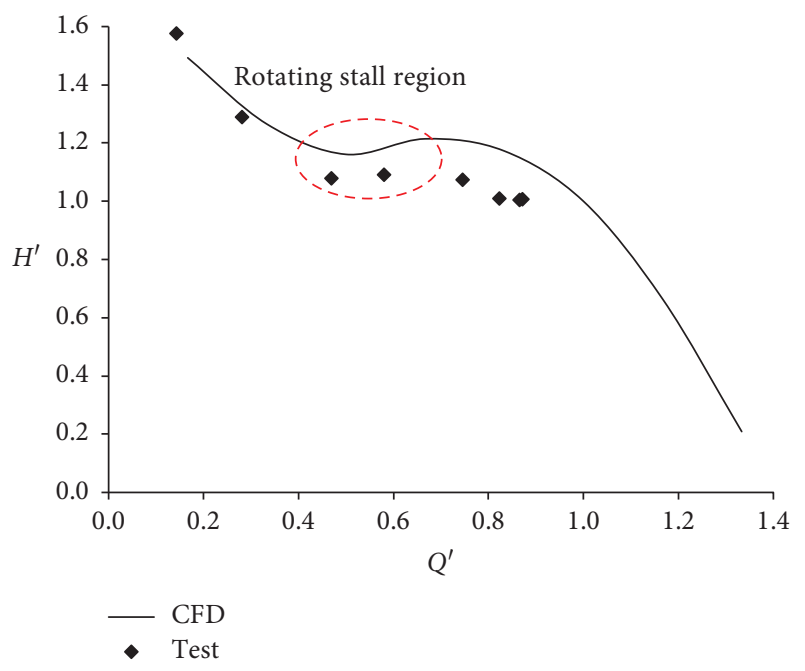

(a)

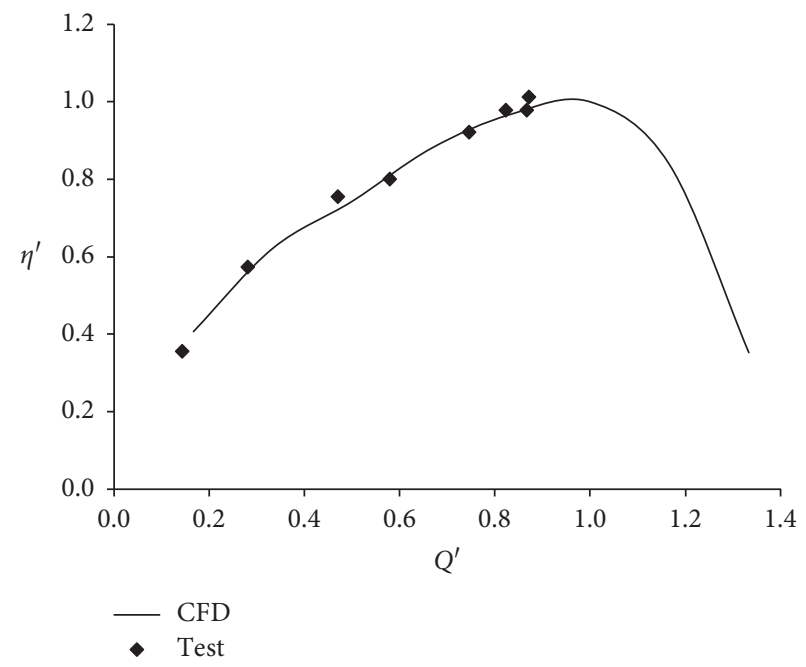

(b)

FIGURE 4: Head and efficiency of CFD and experimental results: (a) $Q^{\prime}-H^{\prime}$ curve; (b) $Q^{\prime}-\eta^{\prime}$ curve.

\section{Research Schemes}

As shown in Figure 4, rotating stall happens when the flow rate $Q^{\prime}$ is between 0.5 and 0.6 . Therefore, a group of grooves is proposed to suppress the rotating stall at the entrance of the impeller shown in Figure 5(a). And the orthogonal experiment schemes are designed.

4.1. Scheme Design. According to the literature [32], the factors and levels of orthogonal experiments should be determined firstly. As shown in Figures 5(b)-5(d), the factors are the geometrical parameters of the groove which are the groove width $W_{\text {groove }}$, the groove number $N_{\text {groove }}$, the groove length $L_{\text {groove, }}$, and the groove depth $D_{\text {groove }}$. For each factor, the number of level is four. The factors and levels of the groove are listed in Table 1. The orthogonal experiment adopted in this paper is an equal level orthogonal experiment. Therefore, $\mathrm{L}_{16}(44)$ orthogonal experiment table is designed in Table 2 based on the method in literature [32].
4.2. Results Analysis. To compare the performance of each scheme, seventeen schemes containing orthogonal experiment schemes and original scheme are numerically calculated. For the waterjet propulsion device, not only the flow rate, head, and efficiency but also the thrust of the device should be paid attention to. Therefore, the four parameters above are selected as evaluation indexes. Regardless of interaction, intuitive analysis is performed with integrated balance method or comprehensive scoring method when multiple evaluation indicators are involved. In this paper, the integrated balance method is utilized to proceed the intuitive analysis. Therefore, the primary factors affecting the performance of device are investigated, and the optimal scheme is obtained.

The serial numbers and the corresponding results of each scheme are listed in Table 3 . To gain the thrust performance, the unit power thrust is introduced. By applying formula (4), the unit power thrust is obtained and written as $F_{P-T}$, and its calculation formula is as follows:

$$
F_{P-T}=\frac{F_{P}}{P_{\text {shaft }}},
$$




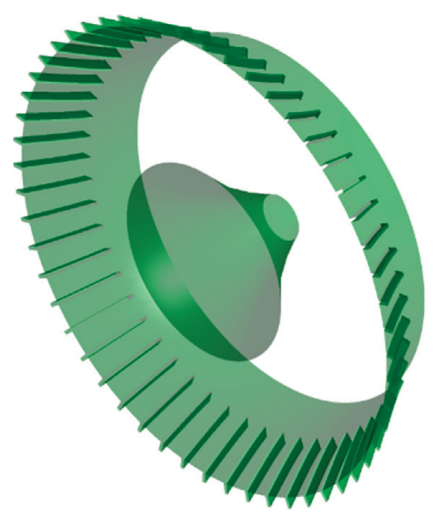

(a)

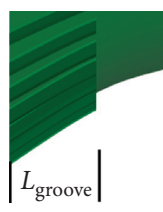

(b)

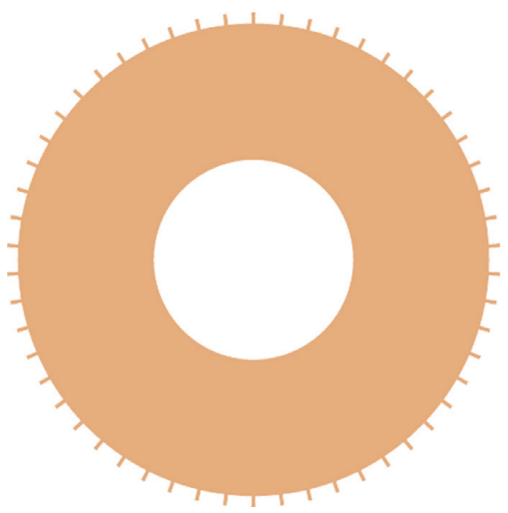

(c)

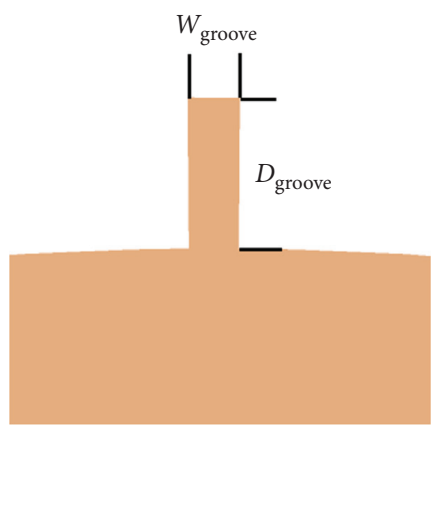

(d)

FIgURE 5: Diagram of groove's location and size: (a) location; (b) length; (c) number; (d) width and depth.

TABLe 1: Factors and levels of groove.

\begin{tabular}{lcccc}
\hline Levels & & Factors & \\
& $\mathrm{A}\left(W_{\text {groove }}\right)$ & $\mathrm{B}\left(N_{\text {groove }}\right)$ & $\mathrm{C}\left(L_{\text {groove }}\right)$ & $\mathrm{D}\left(D_{\text {groove }}\right)$ \\
\hline 1 & $7.75 \times 10^{-3} \mathrm{D}$ & 18 & $7.75 \times 10^{-2} \mathrm{D}$ & $7.75 \times 10^{-3} \mathrm{D}$ \\
2 & $1.55 \times 10^{-2} \mathrm{D}$ & 36 & $1.55 \times 10^{-1} \mathrm{D}$ & $1.55 \times 10^{-2} \mathrm{D}$ \\
3 & $2.32 \times 10^{-2} \mathrm{D}$ & 54 & $2.32 \times 10^{-1} \mathrm{D}$ & $2.32 \times 10^{-2} \mathrm{D}$ \\
4 & $3.10 \times 10^{-2} \mathrm{D}$ & 72 & $3.10 \times 10^{-1} \mathrm{D}$ & $3.10 \times 10^{-2} \mathrm{D}$ \\
\hline
\end{tabular}

TABLE 2: $\mathrm{L}_{16}\left(4^{4}\right)$ orthogonal experiment table.

\begin{tabular}{|c|c|c|c|c|c|c|c|c|}
\hline \multirow{2}{*}{ Schemes } & \multicolumn{4}{|c|}{ Serial numbers } & \multicolumn{4}{|c|}{ Parameters } \\
\hline & A & B & $\mathrm{C}$ & $\mathrm{D}$ & $W_{\text {groove }}$ & $N_{\text {groove }}$ & $L_{\text {groove }}$ & $D_{\text {groove }}$ \\
\hline 1 & 1 & 1 & 1 & 1 & $7.75 \times 10^{-3} \mathrm{D}$ & 18 & $7.75 \times 10^{-2} \mathrm{D}$ & $7.75 \times 10^{-3} \mathrm{D}$ \\
\hline 2 & 1 & 2 & 2 & 2 & $7.75 \times 10^{-3} \mathrm{D}$ & 36 & $1.55 \times 10^{-1} \mathrm{D}$ & $1.55 \times 10^{-2} \mathrm{D}$ \\
\hline 3 & 1 & 3 & 3 & 3 & $7.75 \times 10^{-3} \mathrm{D}$ & 54 & $2.32 \times 10^{-1} \mathrm{D}$ & $2.32 \times 10^{-2} \mathrm{D}$ \\
\hline 4 & 1 & 4 & 4 & 4 & $7.75 \times 10^{-3} \mathrm{D}$ & 72 & $3.10 \times 10^{-1} \mathrm{D}$ & $3.10 \times 10^{-2} \mathrm{D}$ \\
\hline 5 & 2 & 1 & 2 & 3 & $1.55 \times 10^{-2} \mathrm{D}$ & 18 & $1.55 \times 10^{-1} \mathrm{D}$ & $2.32 \times 10^{-2} \mathrm{D}$ \\
\hline 6 & 2 & 2 & 1 & 4 & $1.55 \times 10^{-2} \mathrm{D}$ & 36 & $7.75 \times 10^{-2} \mathrm{D}$ & $3.10 \times 10^{-2} \mathrm{D}$ \\
\hline 7 & 2 & 3 & 4 & 1 & $1.55 \times 10^{-2} \mathrm{D}$ & 54 & $3.10 \times 10^{-1} \mathrm{D}$ & $7.75 \times 10^{-3} \mathrm{D}$ \\
\hline 8 & 2 & 4 & 3 & 2 & $1.55 \times 10^{-2} \mathrm{D}$ & 72 & $2.32 \times 10^{-1} \mathrm{D}$ & $1.55 \times 10^{-2} \mathrm{D}$ \\
\hline 9 & 3 & 1 & 3 & 4 & $2.32 \times 10^{-2} \mathrm{D}$ & 18 & $2.32 \times 10^{-1} \mathrm{D}$ & $3.10 \times 10^{-2} \mathrm{D}$ \\
\hline 10 & 3 & 2 & 4 & 3 & $2.32 \times 10^{-2} \mathrm{D}$ & 36 & $3.10 \times 10^{-1} \mathrm{D}$ & $2.32 \times 10^{-2} \mathrm{D}$ \\
\hline 11 & 3 & 3 & 1 & 2 & $2.32 \times 10^{-2} \mathrm{D}$ & 54 & $7.75 \times 10^{-2} \mathrm{D}$ & $1.55 \times 10^{-2} \mathrm{D}$ \\
\hline 12 & 3 & 4 & 2 & 1 & $2.32 \times 10^{-2} \mathrm{D}$ & 72 & $1.55 \times 10^{-1} \mathrm{D}$ & $7.75 \times 10^{-3} \mathrm{D}$ \\
\hline 13 & 4 & 1 & 4 & 2 & $3.10 \times 10^{-2} \mathrm{D}$ & 18 & $3.10 \times 10^{-1} \mathrm{D}$ & $1.55 \times 10^{-2} \mathrm{D}$ \\
\hline 14 & 4 & 2 & 3 & 1 & $3.10 \times 10^{-2} \mathrm{D}$ & 36 & $2.32 \times 10^{-1} \mathrm{D}$ & $7.75 \times 10^{-3} \mathrm{D}$ \\
\hline 15 & 4 & 3 & 2 & 4 & $3.10 \times 10^{-2} \mathrm{D}$ & 54 & $1.55 \times 10^{-1} \mathrm{D}$ & $3.10 \times 10^{-2} \mathrm{D}$ \\
\hline 16 & 4 & 4 & 1 & 3 & $3.10 \times 10^{-2} \mathrm{D}$ & 72 & $7.75 \times 10^{-2} \mathrm{D}$ & $2.32 \times 10^{-2} \mathrm{D}$ \\
\hline
\end{tabular}

where $F_{P}$ is the thrust of the device in N, $P_{\text {shaft }}$ is the shaft power in $\mathrm{kW}$, and $F_{P-T}$ is the unit power thrust in $\mathrm{N} \cdot \mathrm{kW}^{-1}$.

Since the stall suppression is mainly concerned with the hydraulic performance of the device, the flow coefficient $K_{Q}$, the total head coefficient $K_{\mathrm{HT}}$, and the efficiency $\eta$ of the device are also selected as analysis parameters.

Through the intuitive analysis of each evaluation index, the primary factor and the optimal scheme corresponding to each index are gained and listed in Table 4. In the table, $K_{1}, K_{2}, K_{3}$, and $K_{4}$ are the summation of the analytical indicators at the same level of each factor, and $k_{1}, k_{2}, k_{3}$, and $k_{4}$ are the average values of the analytical indicators at the same level of each factor. The range $R$ is the difference between the maximum and the minimum average values of the analytical indicators at the same level of each factor. The rank of different factors is up to the influence on the analytical indicators. The far left is the most critical factor.

According to Table 4, the range of each parameter for the other factors is normalized by dividing the range of each parameter for factor $\mathrm{A}$ and are, respectively, recorded as $K_{Q^{\prime}}$, $K_{\mathrm{HT}^{\prime}}, \eta^{\prime}$, and $F_{P-T^{\prime}}$. The results are plotted as the range diagram of each parameter for different factors, as shown in Figure 6.

Considering the optimal scheme corresponding to each index is different, each factor should be analyzed separately. 
TABLE 3: Results of each orthogonal experiment scheme.

\begin{tabular}{|c|c|c|c|c|c|c|c|c|}
\hline \multirow{2}{*}{ Schemes } & \multicolumn{4}{|c|}{ Serial numbers } & \multicolumn{4}{|c|}{ Orthogonal experiment results } \\
\hline & A & $\mathrm{B}$ & $\mathrm{C}$ & $\mathrm{D}$ & $K_{Q}$ & $K_{\mathrm{HT}}$ & $\eta(\%)$ & $F_{P-T}\left(\mathrm{~N} \cdot \mathrm{kW}^{-1}\right)$ \\
\hline 1 & 1 & 1 & 1 & 1 & 0.0791 & 0.0743 & 72.69 & 45.39 \\
\hline 2 & 1 & 2 & 2 & 2 & 0.0802 & 0.0743 & 73.45 & 49.12 \\
\hline 3 & 1 & 3 & 3 & 3 & 0.0801 & 0.0740 & 73.18 & 48.79 \\
\hline 4 & 1 & 4 & 4 & 4 & 0.0811 & 0.0741 & 73.93 & 52.19 \\
\hline 5 & 2 & 1 & 2 & 3 & 0.0797 & 0.0742 & 73.09 & 47.62 \\
\hline 6 & 2 & 2 & 1 & 4 & 0.0811 & 0.0739 & 74.08 & 52.41 \\
\hline 7 & 2 & 3 & 4 & 1 & 0.0798 & 0.0743 & 72.89 & 47.59 \\
\hline 8 & 2 & 4 & 3 & 2 & 0.0811 & 0.0742 & 74.10 & 52.21 \\
\hline 9 & 3 & 1 & 3 & 4 & 0.0805 & 0.0740 & 73.56 & 50.25 \\
\hline 10 & 3 & 2 & 4 & 3 & 0.0813 & 0.0739 & 73.78 & 52.74 \\
\hline 11 & 3 & 3 & 1 & 2 & 0.0814 & 0.0741 & 74.25 & 53.12 \\
\hline 12 & 3 & 4 & 2 & 1 & 0.0806 & 0.0744 & 73.75 & 50.46 \\
\hline 13 & 4 & 1 & 4 & 2 & 0.0800 & 0.0740 & 72.97 & 48.35 \\
\hline 14 & 4 & 2 & 3 & 1 & 0.0800 & 0.0744 & 73.15 & 48.36 \\
\hline 15 & 4 & 3 & 2 & 4 & 0.0855 & 0.0735 & 77.25 & 68.35 \\
\hline 16 & 4 & 4 & 1 & 3 & 0.0854 & 0.0736 & 77.33 & 68.12 \\
\hline
\end{tabular}

For factor $\mathrm{A}, \mathrm{A}_{4}$ holds the optimal flow coefficient, efficiency, and unit power thrust. Especially for the two key parameters of efficiency and unit power thrust, factor A is the primary factor. By comparing the ranges of the head coefficient, it is found that the deviation between the ranges of the factor $\mathrm{A}$ is small, so $\mathrm{A}_{4}$ is selected for the factor $\mathrm{A}$. For factor $\mathrm{B}, \mathrm{B}_{4}$ possesses the most excellent flow coefficient, efficiency, and unit power thrust. The influence of factor $B$ is inferior to factor $\mathrm{A}$. Therefore, $\mathrm{B}_{4}$ is selected for factor $\mathrm{B}$. For factor $\mathrm{C}$, the range of the flow coefficient, efficiency, and unit power thrust is the last position and the range of head coefficient is the second last position. So factor $\mathrm{C}$ has less impact on the performance of the device. $\mathrm{C}_{1}$ encompasses the optimal flow coefficient and unit power thrust. Considering the little difference of the range for the efficiency and head coefficient, $\mathrm{C}_{1}$ is chosen for factor C. For factor D, $\mathrm{D}_{4}$ owns the maximum low coefficient, efficiency, and unit power thrust. But the optimal head coefficient is $D_{1}$, and the factor $D$ is the primary factor. However, the waterjet propulsion system is to generate the maximum thrust as much as possible with high efficiency. Therefore, $\mathrm{D}_{4}$ is chosen for factor D.

It can be concluded that the order of influence of the four factors on the performance of the waterjet propulsion pump device is A, B, D, and C, that is, the groove width, the number of grooves, the depth of the groove, and the length of the groove. The best combination of each parameter is $\mathrm{A}_{4} \mathrm{~B}_{4} \mathrm{C}_{1} \mathrm{D}_{4}$. The specific parameters of the groove are $7.75 \times 10^{-2} \mathrm{D}$ in length, $3.10 \times 10^{-2} \mathrm{D}$ in width, $3.10 \times 10^{-2} \mathrm{D}$ in depth, and 72 in number of grooves. Among the 16 schemes, scheme 16 is closest to the above parameters. Scheme 16 was chosen as the final scheme to suppress rotating stall.

\section{Analysis of Stall Suppression Effect}

5.1. Hydraulic Characteristics. Different flow rate conditions of the selected scheme were calculated, and the data of the whole flow field were obtained. The comparison between the scheme and the original scheme was made to analyze the effect of the scheme on stall suppression. Based on the results under the optimal operating condition of the original scheme, the flow rate, head, and efficiency of the selected scheme and the original scheme were normalized, and the hydraulic performance of the two schemes is plotted in Figure 7. The optimal condition, the condition entering the rotating stall area, and the condition at the valley bottom of the rotating stall area are three characteristic conditions, which are $1.0 Q^{\prime}, 0.67 Q^{\prime}$, and $0.5 Q^{\prime}$, respectively. After the groove was added, it was found that the head rose under $0.5 \mathrm{Q}^{\prime}$ condition and then decreased under $0.67 Q^{\prime}$ condition, the positive slope area of the $Q^{\prime}-H^{\prime}$ curve disappeared, the hydraulic instability area was eliminated, the rotating stall was well suppressed, and the $Q^{\prime}-H^{\prime}$ curve basically coincides under the large flow rate condition. Comparing the $Q^{\prime}-\eta^{\prime}$ curve of the two schemes, it is found that the overall efficiency of the selected scheme is lower than that of the original scheme. Because the geometrical shape of the groove changes greatly, the hydraulic loss, especially the part hydraulic loss, increases greatly, which results in the increase in the hydraulic loss of the transition section at the impeller inlet. In this paper, when calculating the efficiency of the propulsion pump, the inlet section is located at the front of the transition section at the impeller inlet, and the increase in the hydraulic loss of the transition section at the inlet will be reflected in the calculation results. One of the hazards of the rotating stall is that it may induce the resonance of the whole device, causing damage to the operation of the unit. Therefore, although the scheme may cause a decrease in the efficiency of the device, it can better ensure the safe and stable operation of the device.

In order to explore the energy change of waterjet propulsion pump device in the process of flow under different working conditions, the total pressure coefficient $K_{\mathrm{HTP}}$ is taken as the parameter to evaluate the energy change: 


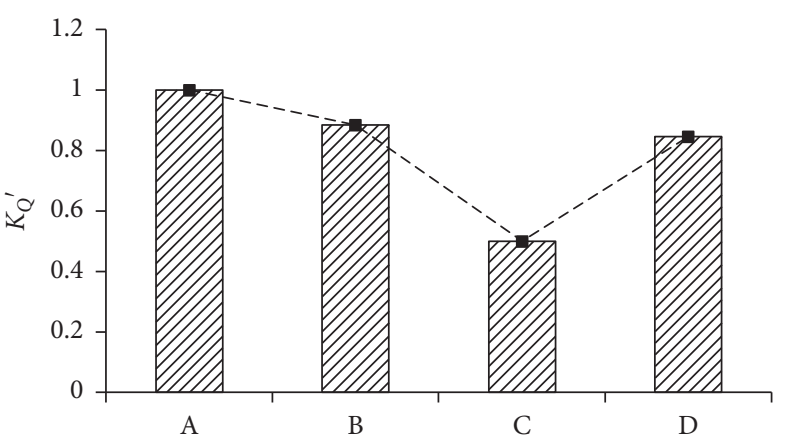

(a)

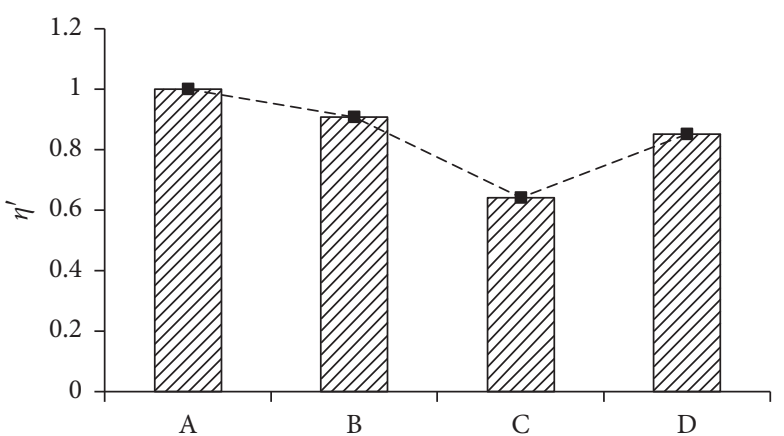

(c)

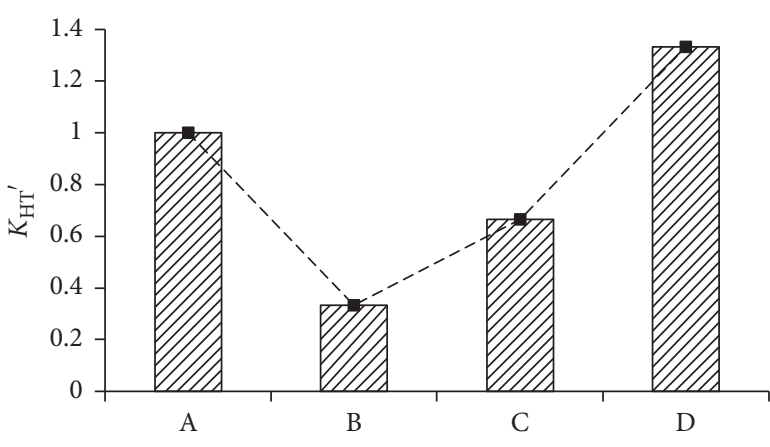

(b)

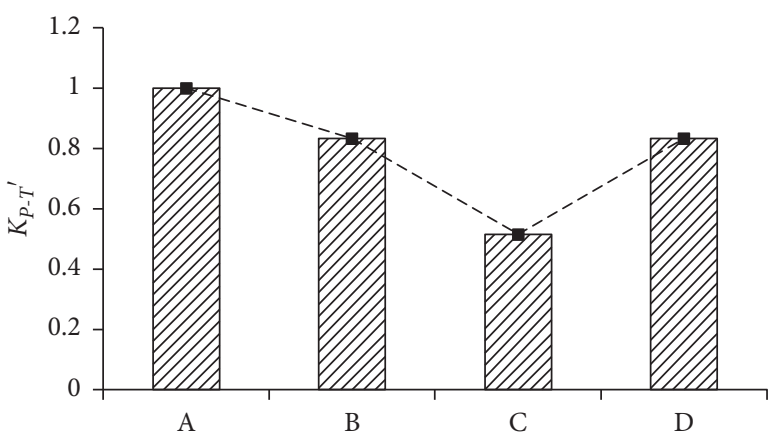

(d)

FIgURE 6: Range diagram of each parameter for different factors: (a) $K_{Q^{\prime}}$; (b) $K_{\mathrm{HT}^{\prime}}$; (c) $\eta^{\prime}$; (d) $F_{P-T^{\prime}}$.

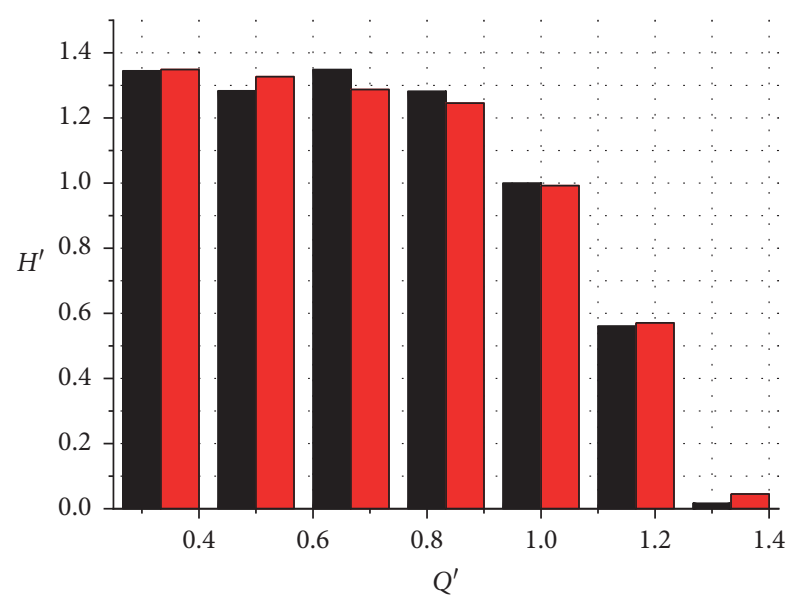

Original scheme Selected scheme

(a)

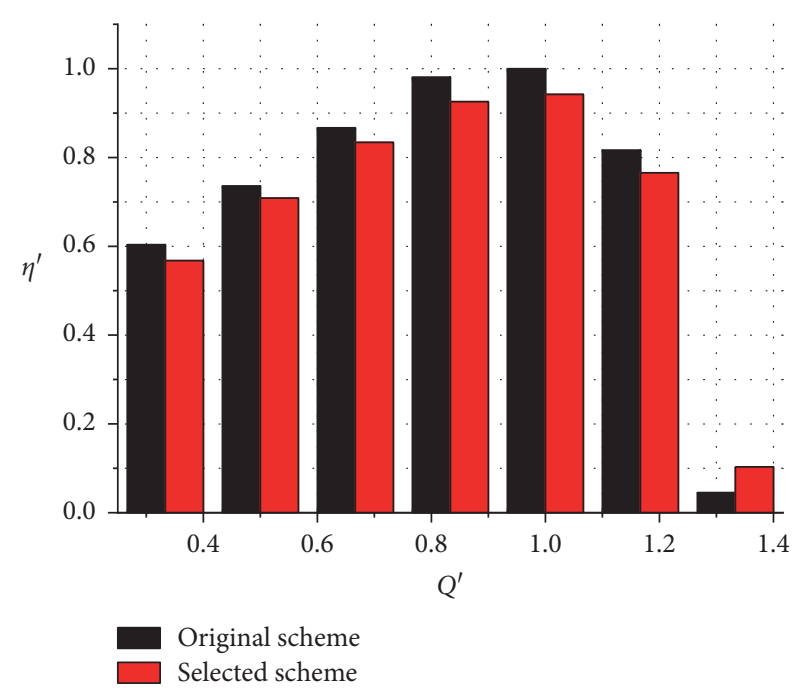

(b)

Figure 7: Hydraulic performance of the selected scheme and the original scheme: (a) $Q^{\prime}-H^{\prime}$ curve; (b) $Q^{\prime}-\eta^{\prime}$ curve.

The upward trend is due to the work done by the propulsion pump to the water, which makes the total pressure of the water increase. The last downward trend is due to the hydraulic loss in the nozzle. Through the total pressure coefficient between section 3-3 and section 4-4 and section 2-2 and section 3-3, the total pressure coefficient difference between the inlet transition section of impeller and the inlet and outlet of propulsion pump section can be calculated. Under the same working condition, the total pressure coefficient difference of the impeller inlet transition section of the selected scheme increases obviously. With the increase in flow rate, the difference becomes larger. Under the $1.0 Q^{\prime}$ working condition, the total pressure coefficient difference of the selected scheme is 6.4 times of the original scheme, which is the reason why the efficiency of the propulsion pump device decreases under the optimal working condition. Under the working condition of $0.5 Q^{\prime}$, the head of propulsion pump increases. Under the working condition of 


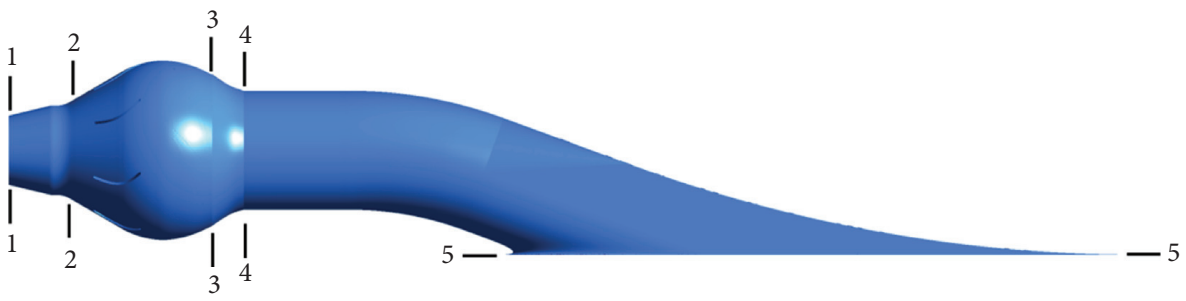

FIGURE 8: Schematic diagram of sections distribution in the device.

TABLE 5: Total pressure coefficient on each section.

\begin{tabular}{llcrrrrr}
\hline & & Original scheme & \multicolumn{3}{c}{ Selected scheme } \\
& & $0.5 Q^{\prime}$ & $0.67 Q^{\prime}$ & $Q^{\prime}$ & $0.5 Q^{\prime}$ & $0.67 Q^{\prime}$ & $Q^{\prime}$ \\
\hline & Section 5-5 & 0.2970 & 0.2956 & 0.2950 & 0.2979 & 0.2977 & 0.2966 \\
Total pressure coefficient & Section 4-4 & 0.2884 & 0.2894 & 0.2908 & 0.2839 & 0.2882 & 0.2908 \\
& Section 3-3 & 0.2855 & 0.2876 & 0.2903 & 0.2806 & 0.2857 & 0.2876 \\
& Section 2-2 & 0.3665 & 0.3712 & 0.3515 & 0.3652 & 0.3665 & 0.3515 \\
& Section 1-1 & 0.3580 & 0.3630 & 0.3453 & 0.3562 & 0.3582 & 0.3446 \\
\hline
\end{tabular}
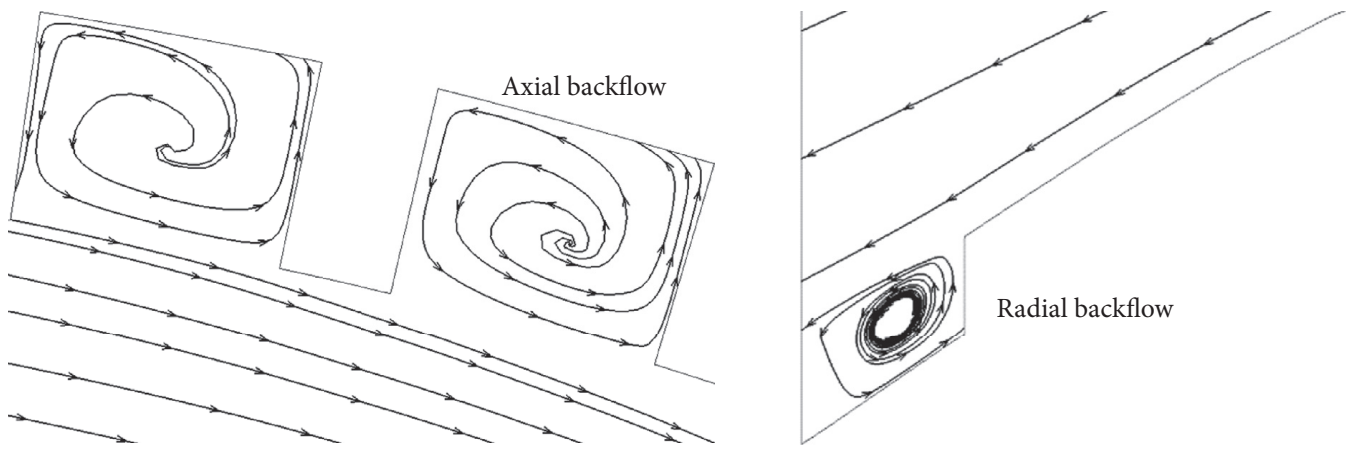

FIGURE 9: Streamlines in groove.

$0.67 Q^{\prime}$, the head of propulsion pump decreases. Under the $1.0 Q^{\prime}$ condition, the head of propulsion pump increases.

5.2. Flow Characteristics. The cross section and longitudinal section are selected near the passage outlet. Figure 9 shows the streamline distribution in the grooves of these two sections under the working condition of $0.5 Q^{\prime}$. There are axial and radial backflow in the groove. This is because the circumferential velocity of the groove is large. After being blocked by the groove, the flow near the wall rolls in the groove, and the rotating flow before the impeller inlet is weakened.

In order to explore the vorticity field distribution at the outlet of the inlet passage, the calculation formulas of the vorticity field are shown in

$$
\begin{aligned}
& \omega_{x}=d d y\left(v_{z}\right)-d d z\left(v_{y}\right), \\
& \omega_{y}=d d y\left(v_{x}\right)-d d z\left(v_{z}\right), \\
& \omega_{z}=d d y\left(v_{y}\right)-d d z\left(v_{x}\right), \\
& \omega=\sqrt{\omega_{x}^{2}+\omega_{y}^{2}+\omega_{z}^{2}}
\end{aligned}
$$

where $v_{x}, v_{y}$, and $v_{z}$ are the velocity component in $x, y$, and $z$ directions, respectively, and $\omega_{x}, \omega_{y}, \omega_{z}$, and $\omega$ are the vorticity and total vorticity in $x, y$, and $z$ directions, respectively.

Figure 10 shows the calculation results of the total vorticity on the cross section of the original scheme and the selected scheme. After comparison, it is found that the vorticity outside the cross section of the original scheme is large, and the vorticity in the main flow area is relatively small, but the distribution is not uniform. When the grooves are added, the vorticity at the inlet of the grooves is large and the vorticity gradient in the main flow area changes uniformly.

In order to further explore the flow characteristics in the impeller, the two-dimensional streamlines on the blades of the original scheme and the selected schemes under the working conditions of $0.5 Q^{\prime}, 0.67 Q^{\prime}$, and $1.0 Q^{\prime}$ are drawn in Figures 11-13, respectively. It is found that, under the condition of $0.5 Q^{\prime}$, there are surface vortices on the blades of the original scheme and the selected scheme. The vortex is located at the trailing edge of the suction surface, and the center of the vortex core is at the span of 0.65 . The vortex is observed on five blades of the original scheme and four blades of the selected scheme, which shows that the 


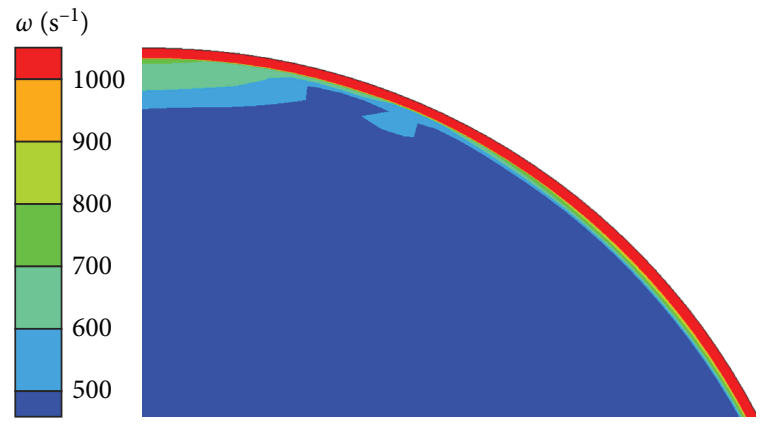

(a)
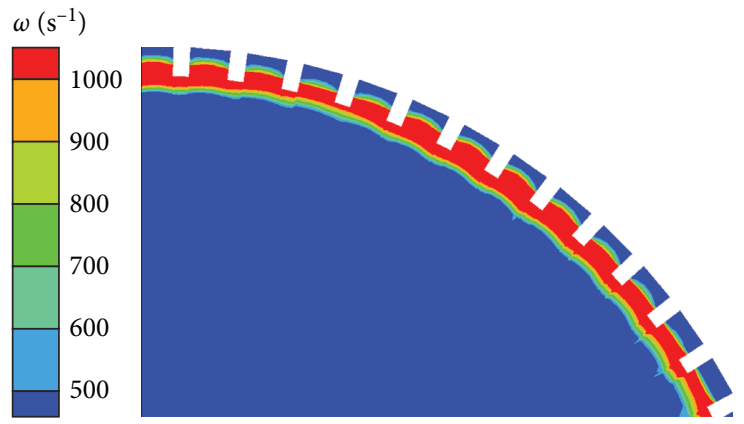

(b)

FIGURE 10: Diagram of vorticity: (a) original scheme; (b) selected scheme.

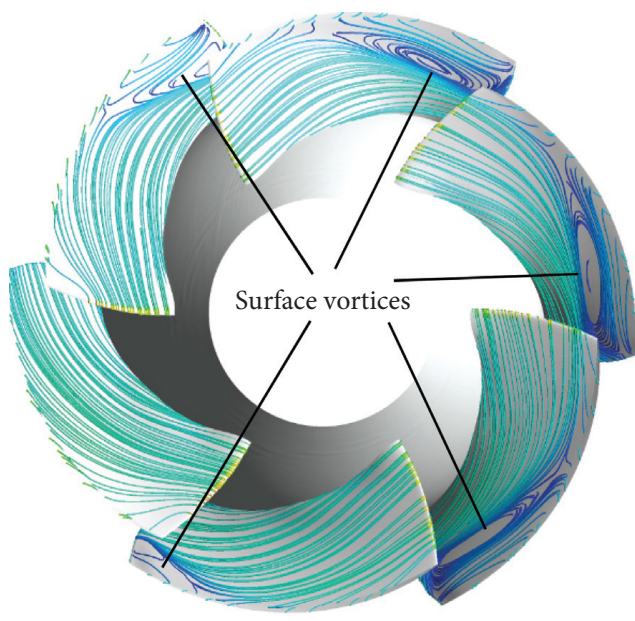

(a)

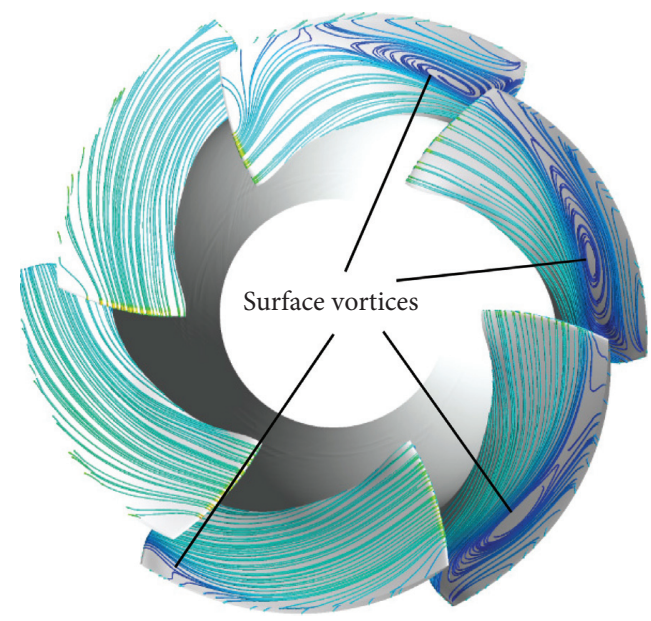

(b)

Figure 11: Streamlines on blade under $0.5 Q^{\prime}$ condition: (a) original scheme; (b) selected scheme.

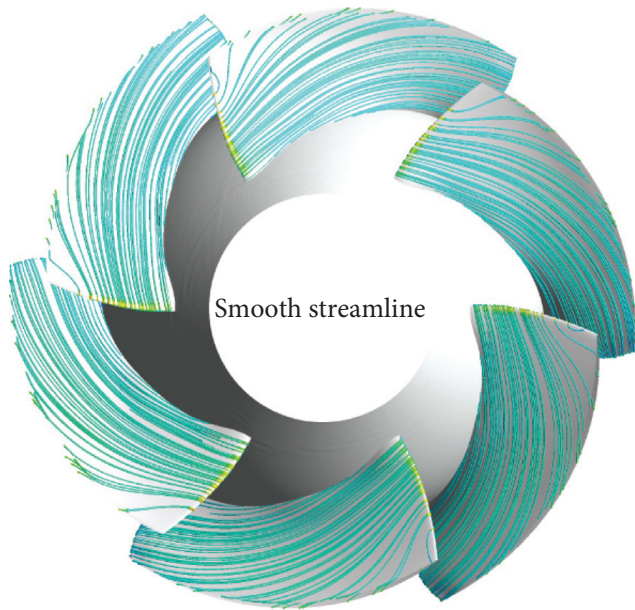

(a)

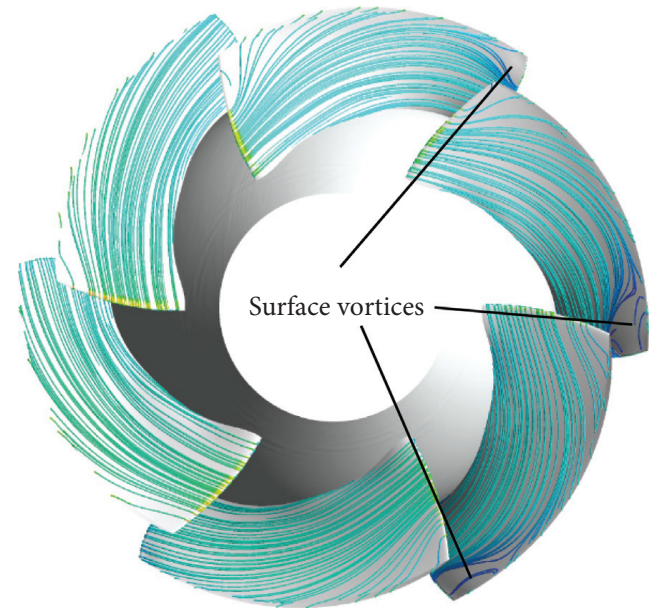

(b)

FIgURE 12: Streamlines on blade under $0.67 Q^{\prime}$ condition: (a) original scheme; (b) selected scheme.

installation of grooves can improve the flow characteristics of the blades to a certain extent. Under the condition of 0.67 $Q^{\prime}$, part of the streamline at the leading edge of the blade in the original scheme flows to the top of the blade and part flows to the trailing edge of the blade, which is relatively smooth. The streamline trend at the inlet of the blade in the selected scheme is basically the same as that in the original scheme, but the flow pattern on the surface of the blade is 


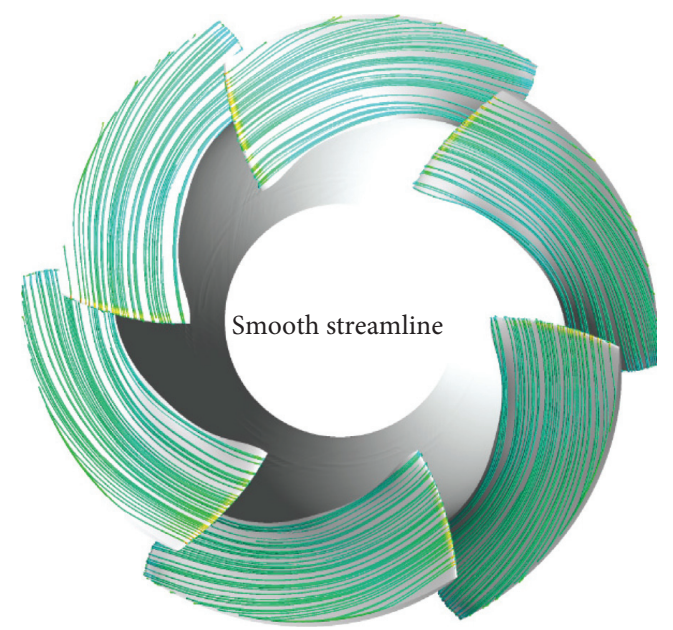

(a)

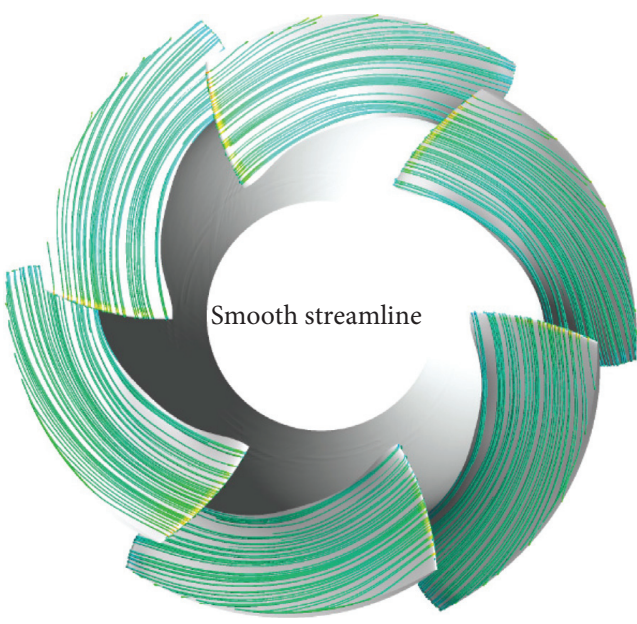

(b)

Figure 13: Streamlines on blade under $1.0 Q^{\prime}$ condition: (a) original scheme; (b) selected scheme.

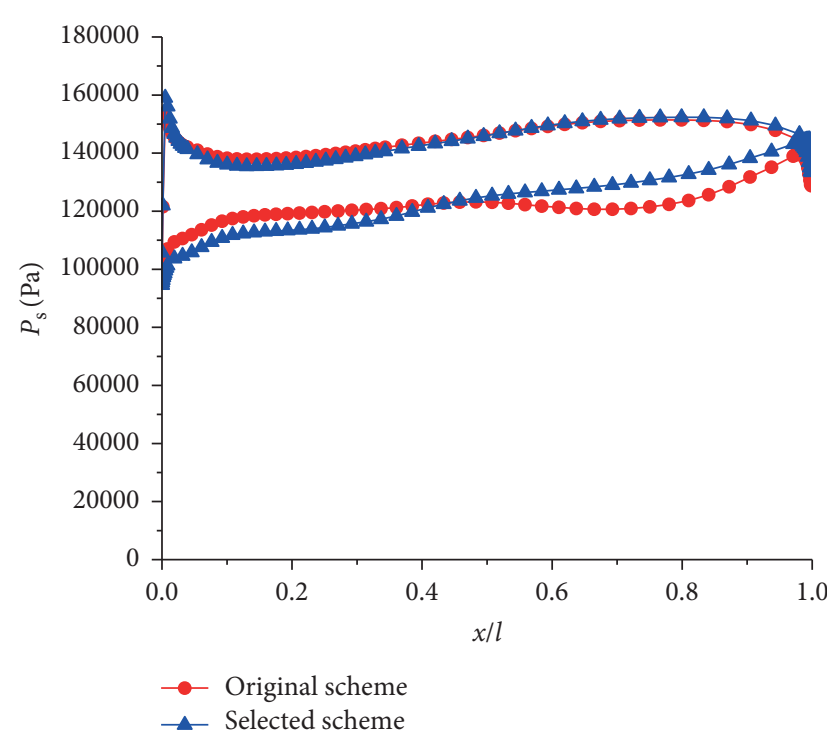

(a)

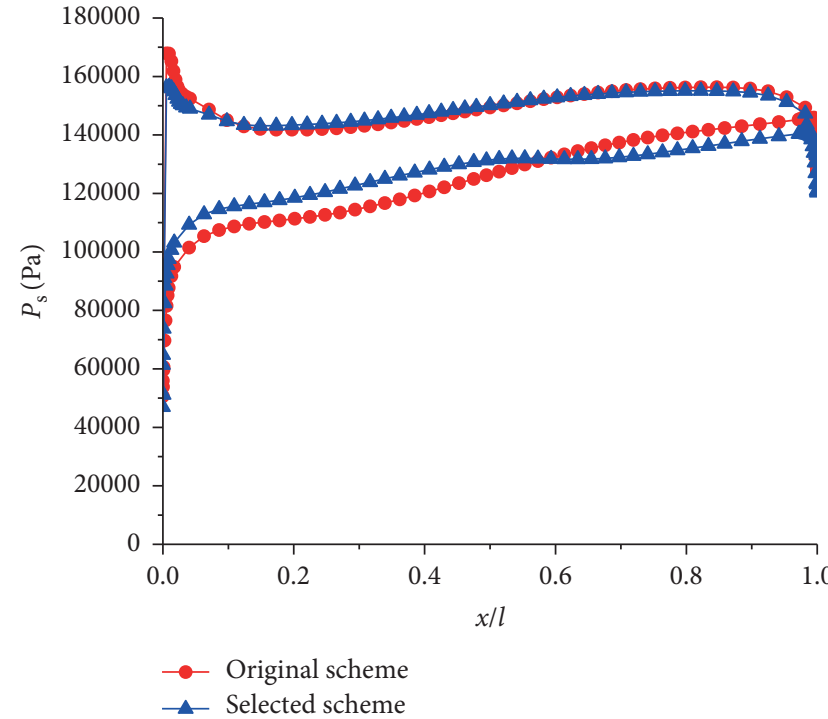

(b)

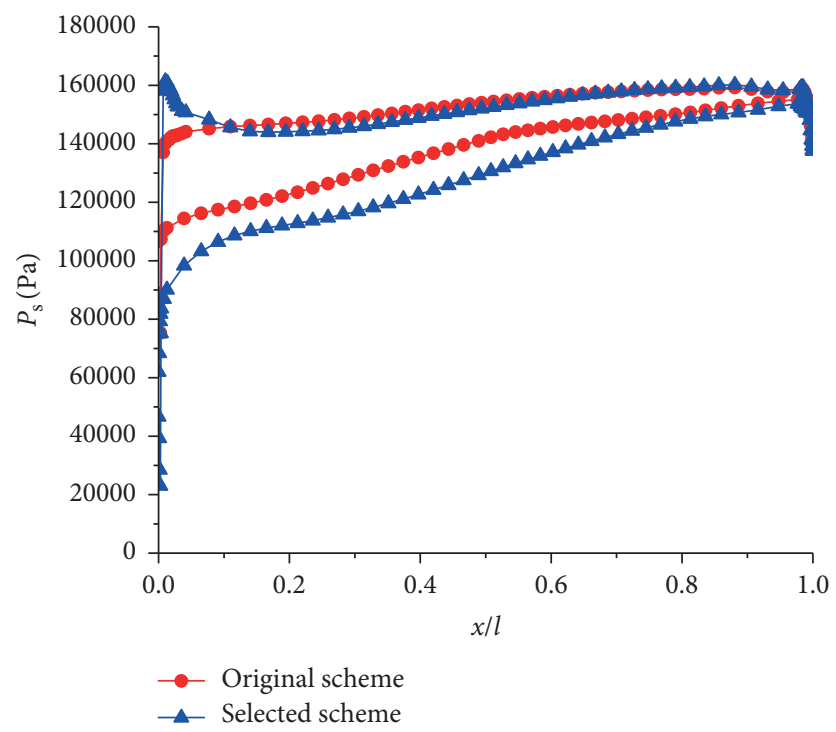

(c)

Figure 14: Static pressure distribution on each span under $0.5 Q^{\prime}$ condition: (a) $\operatorname{span}=0.1$; (b) $\operatorname{span}=0.65$; (c) $\operatorname{span}=0.96$. 


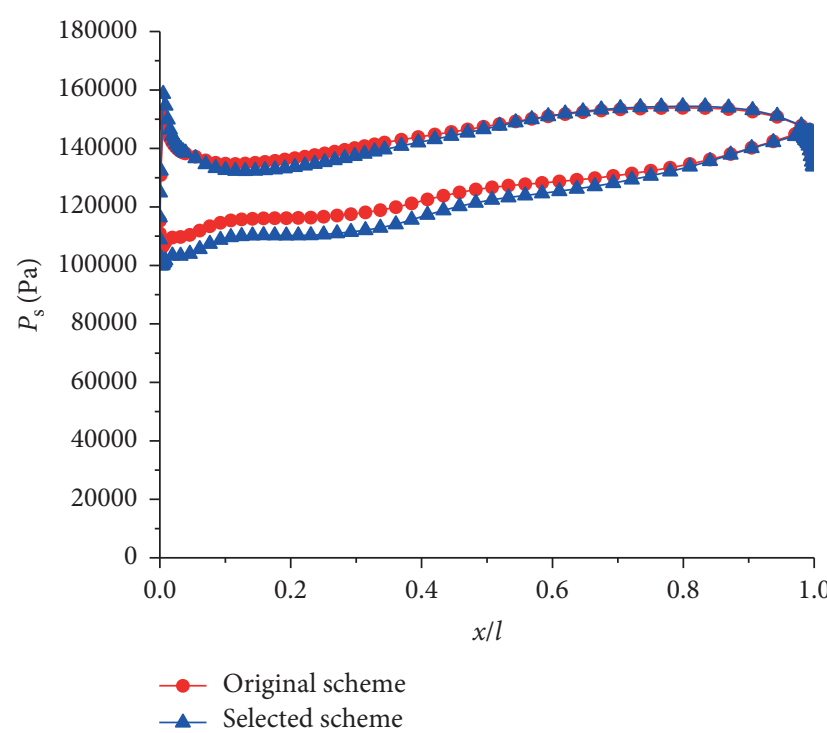

(a)

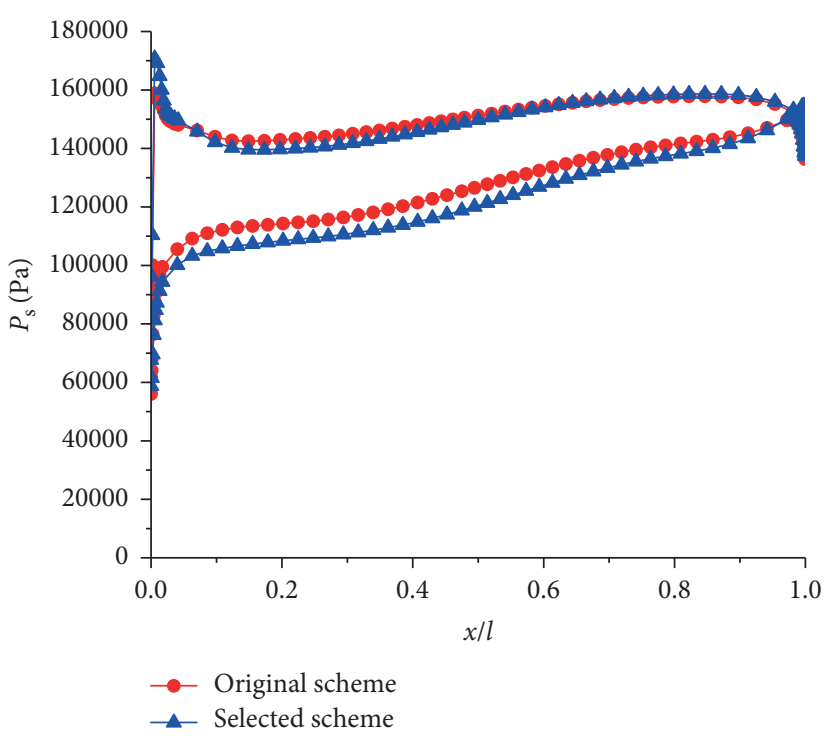

(b)

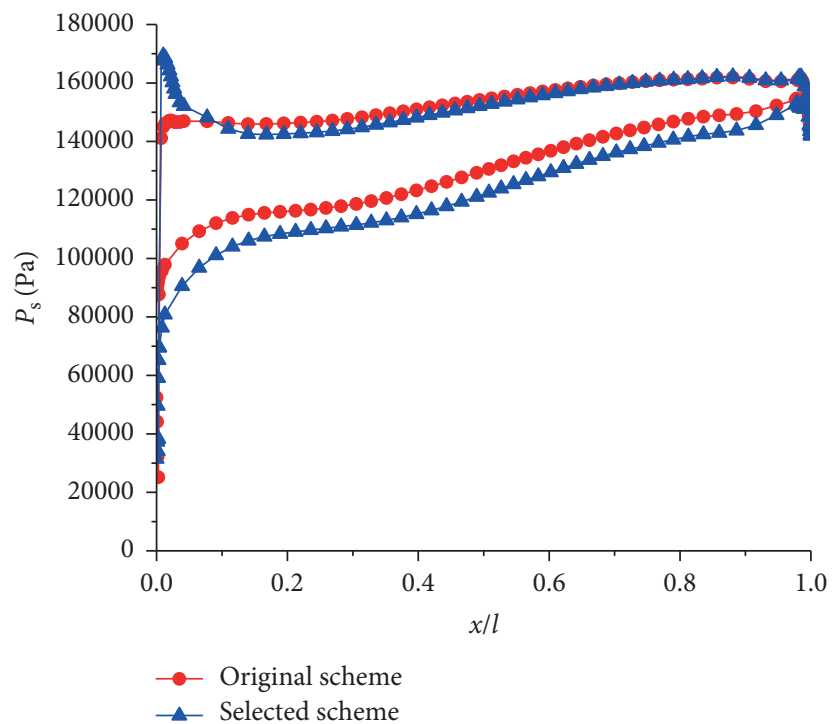

(c)

FiguRe 15: Static pressure distribution on each span under $0.67 Q^{\prime}$ condition: (a) $\operatorname{span}=0.1$; (b) $\operatorname{span}=0.65$; (c) $\operatorname{span}=0.96$.

poor, and a small range of surface vortices can be observed at the trailing edge of some blades. Under the working condition of $1.0 Q^{\prime}$, the streamlines on each blade of the original scheme and the selected scheme are relatively smooth.

Under the working condition of $0.5 Q^{\prime}$, the head of the selected scheme is higher than that of the original scheme, and the flow pattern on the blade is improved. Under the working condition of $0.67 Q^{\prime}$, the head of the selected scheme is decreased, and the streamline on the blade is not smooth as the original scheme. Under the working condition of 1.0 $Q^{\prime}$, the head of the two schemes is not much different, and the flow pattern on the blade is smooth.

The hydraulic performance shows that, under the working condition of $0.5 Q^{\prime}$, that is, at the valley point of the rotating stall region, the head of the selected scheme is significantly increased, while under the condition of $0.67 Q^{\prime}$, the head of the selected scheme is significantly decreased. In order to find out the reason of this change, the static pressure distribution on different spans near the hub $(\operatorname{span}=0.1)$, near the center $(\operatorname{span}=0.65)$, and near the shroud ( $\operatorname{span}=0.96$ ) under these two conditions and optimal condition is compared, and Figures 14-16 are drawn.

Due to the complex and unsteady flow such as the vortex [33] in the blade to blade passage, the static pressure load on the suction and pressure side of each blade is nonidentical under the working condition of $0.5 Q^{\prime}$. Therefore, the static pressure load on the representative blade is analyzed in this paper. The static pressure of the pressure side on $\operatorname{span}=0.65$ and 0.96 of the original scheme is slightly higher than that of the selected scheme. On span $=0.1$, the static pressure on the pressure side is lower than that of the selected scheme at $x /$ 


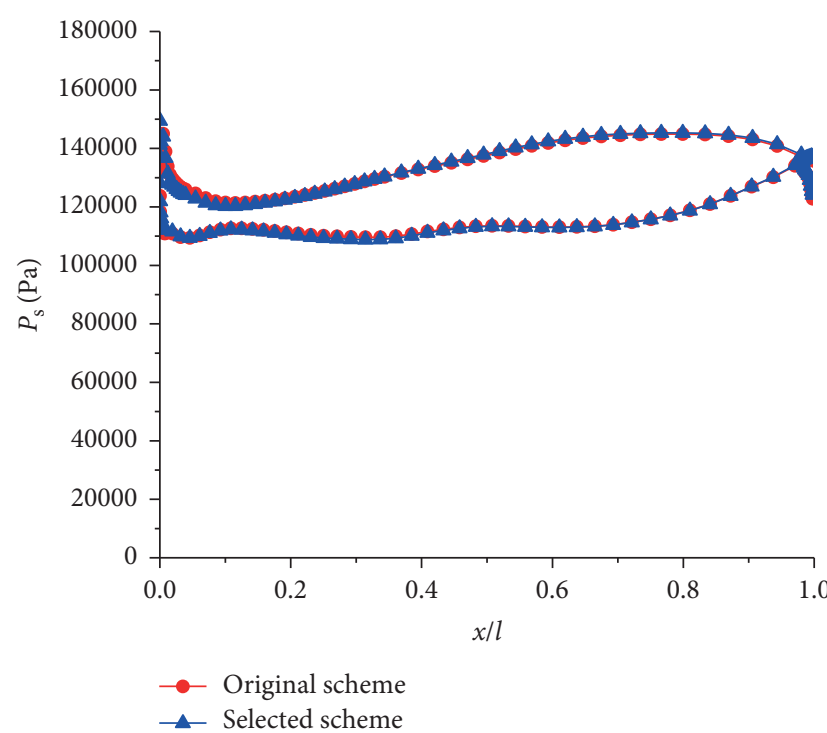

(a)

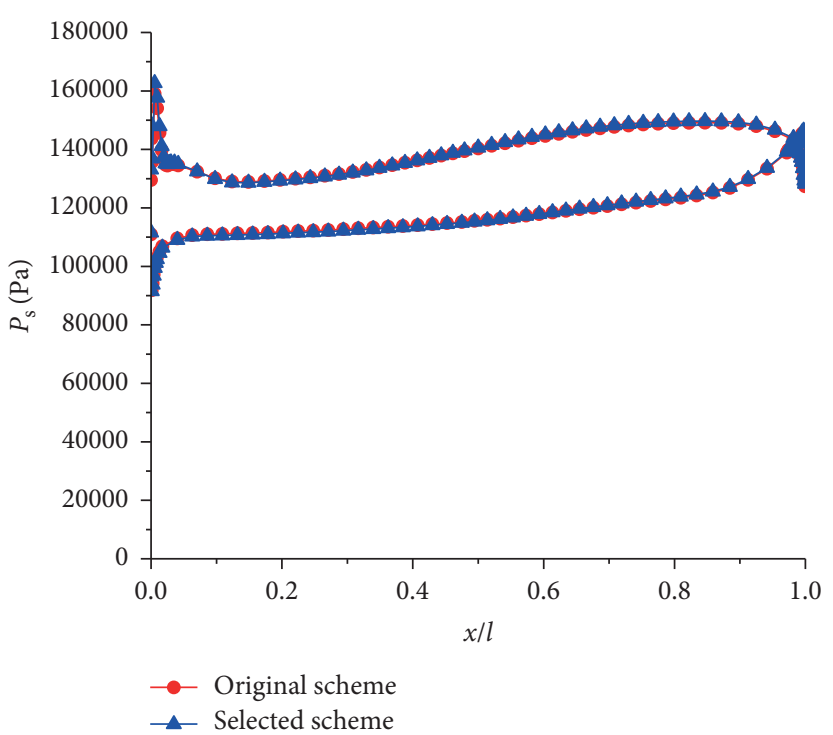

(b)

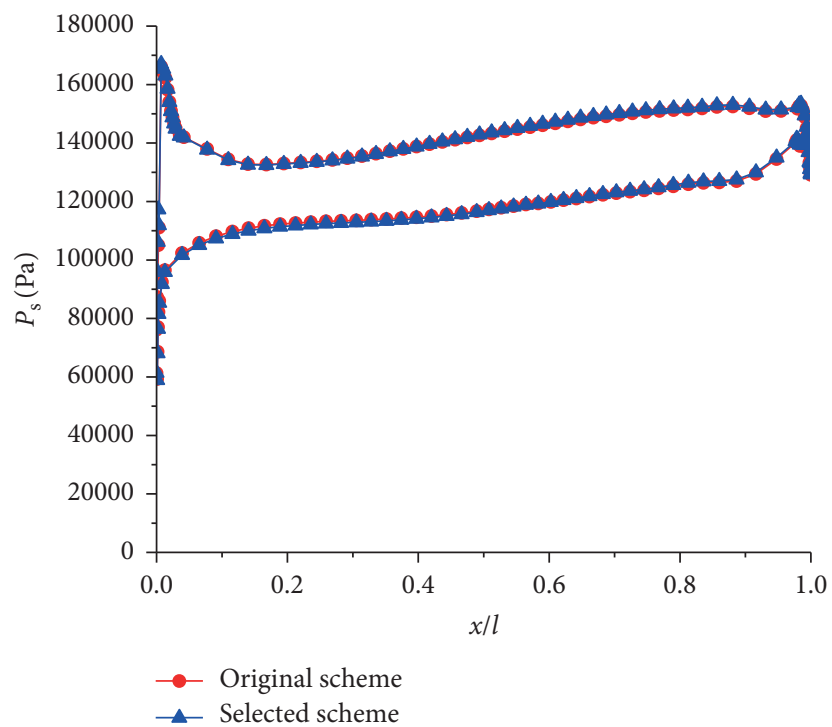

(c)

Figure 16: Static pressure distribution on each span under $1.0 Q^{\prime}$ condition: (a) $\operatorname{span}=0.1$; (b) $\operatorname{span}=0.65$; (c) $\operatorname{span}=0.96$.

$l<0.5$ but is basically consistent with that of the selected scheme at $0.5<x / l<1$. The static pressure on the suction side of each scheme is greater than the selected scheme, especially at $x / l<0.8$ on span $=0.96$, and this trend is more obvious. The static pressure difference between the pressure side and suction side on $\operatorname{span}=0.1$ and 0.96 is apparently lower than that of the selected scheme. And the static pressure between the pressure side and suction side on span $=0.65$ is slightly lower than the selected scheme. Since the work done by the blade is mainly related to the static pressure difference between the suction surface and the pressure surface, the larger the static pressure difference is, the stronger the work done by the blade is. Therefore, the head of the selected scheme is obviously increased under this working condition.

Under the working condition of $0.67 Q^{\prime}$, the waterjet propulsion device starts to enter the rotating stall region. The static pressure on the pressure side of the original scheme is higher than that of the selected scheme. The static pressure of the suction side at $x / l<0.4$ on span $=0.1$ and 0.65 is identical with that of the selected scheme but is slightly higher than that of the selected scheme at $0.4<x / l<1$. The static pressure of the suction side is lower than the selected scheme at $0.2<x / l<0.6$ on span $=0.96$ but is basically the same as the selected scheme at $x / l<0.2$ and $0.6<x / l<1$. On the whole, the static pressure difference between the pressure side and suction side of each turbo span surface is greater than the selected scheme. Therefore, the head of the selected scheme decreases.

Under the working condition of $1.0 Q^{\prime}$, the static pressure distribution on the suction surface and pressure surface of the original scheme and the selected scheme is almost the same, the pressure decrease on both sides is not 

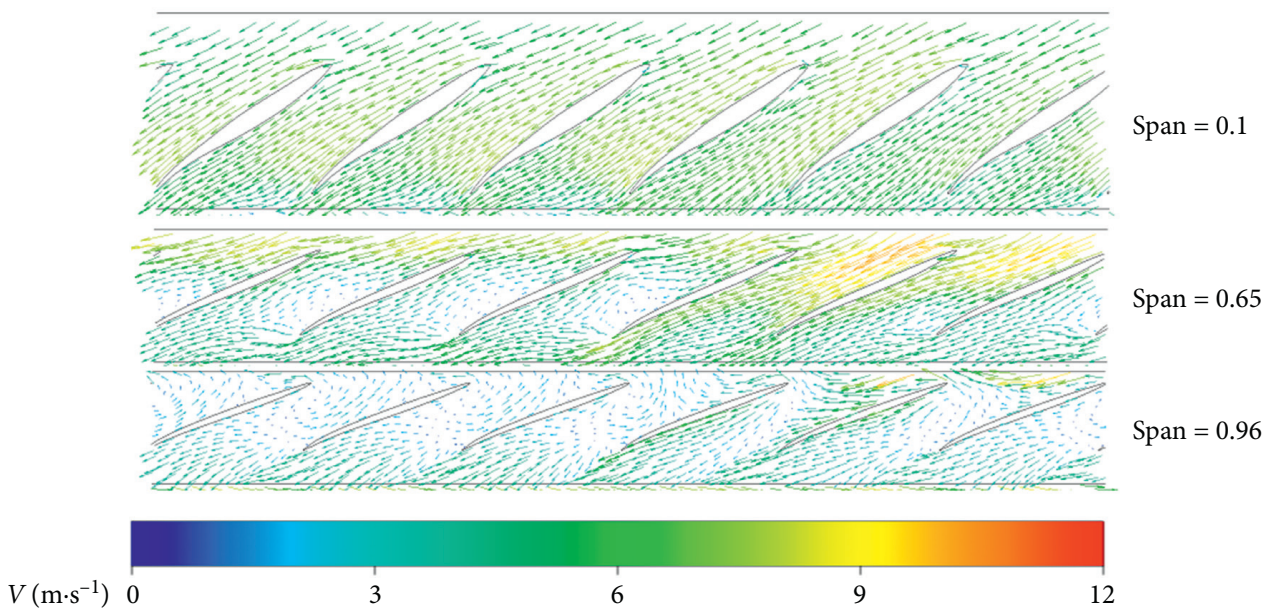

(a)

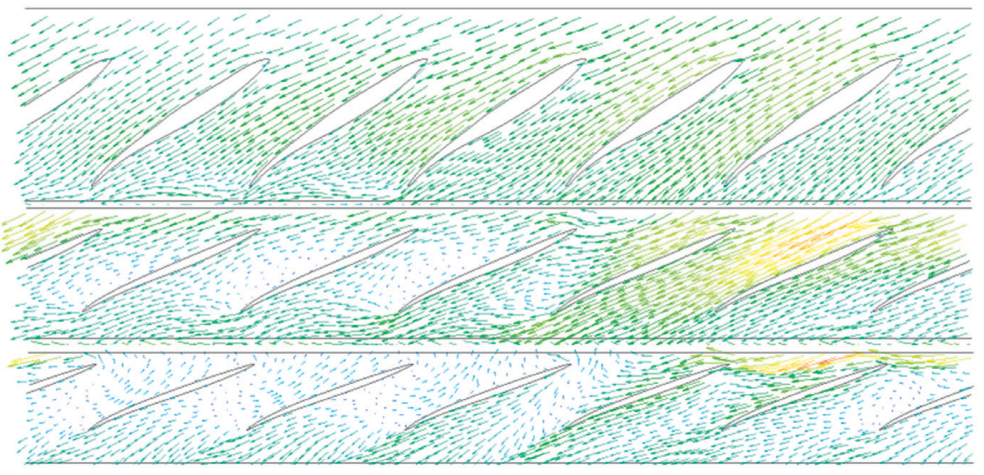

Span $=0.1$

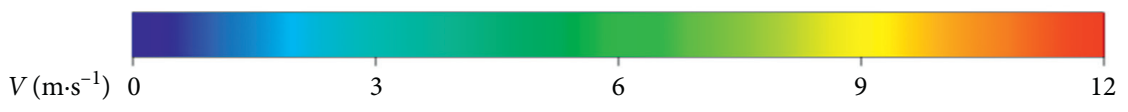

(b)

FIGURE 17: Velocity vector on each span of original scheme and selected scheme under $0.5 Q^{\prime}$ condition: (a) original scheme; (b) selected scheme.

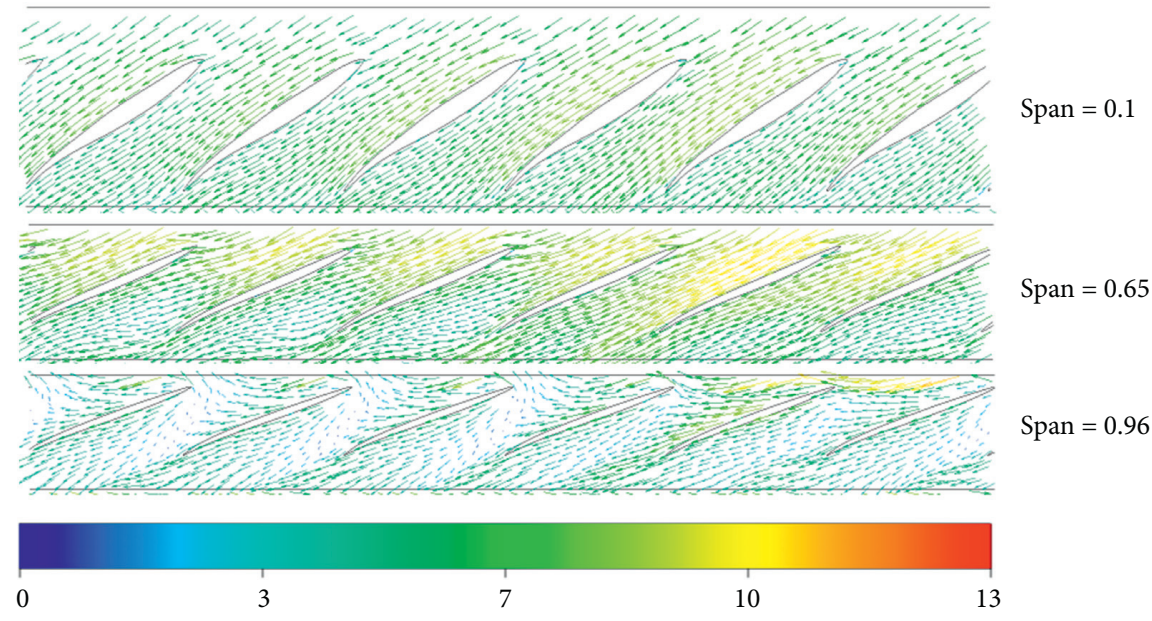

(a)

Figure 18: Continued. 

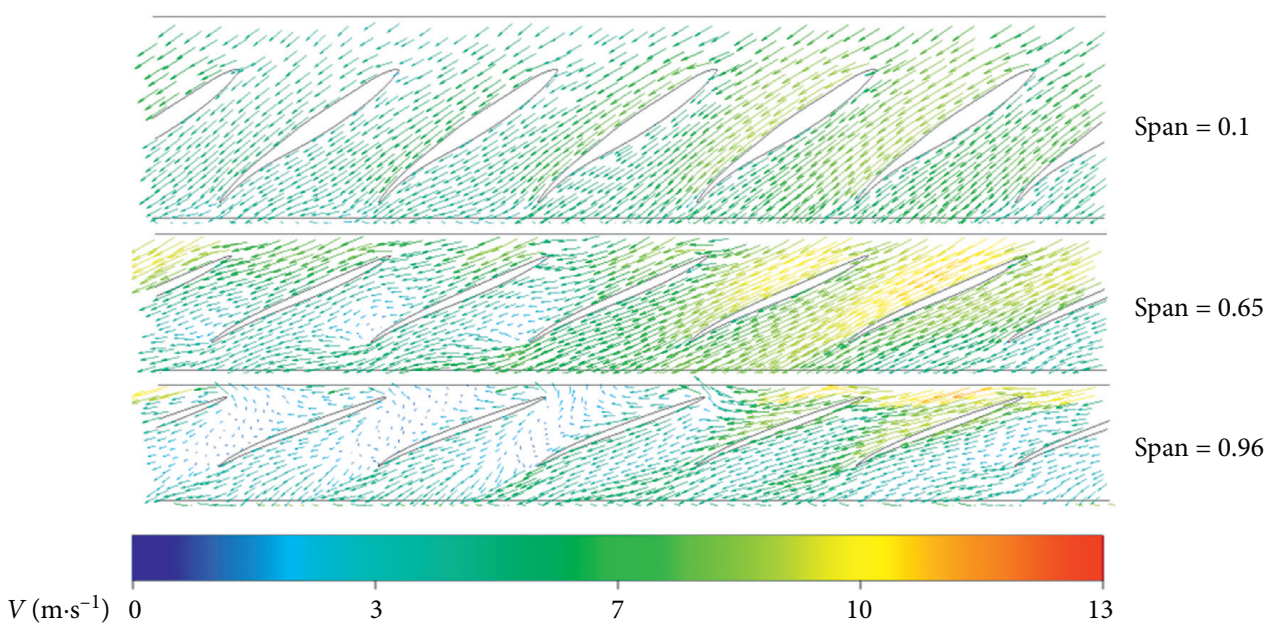

(b)

FIGURE 18: Velocity vector on each span of original scheme and selected scheme under $0.67 Q^{\prime}$ condition: (a) original scheme; (b) selected scheme.
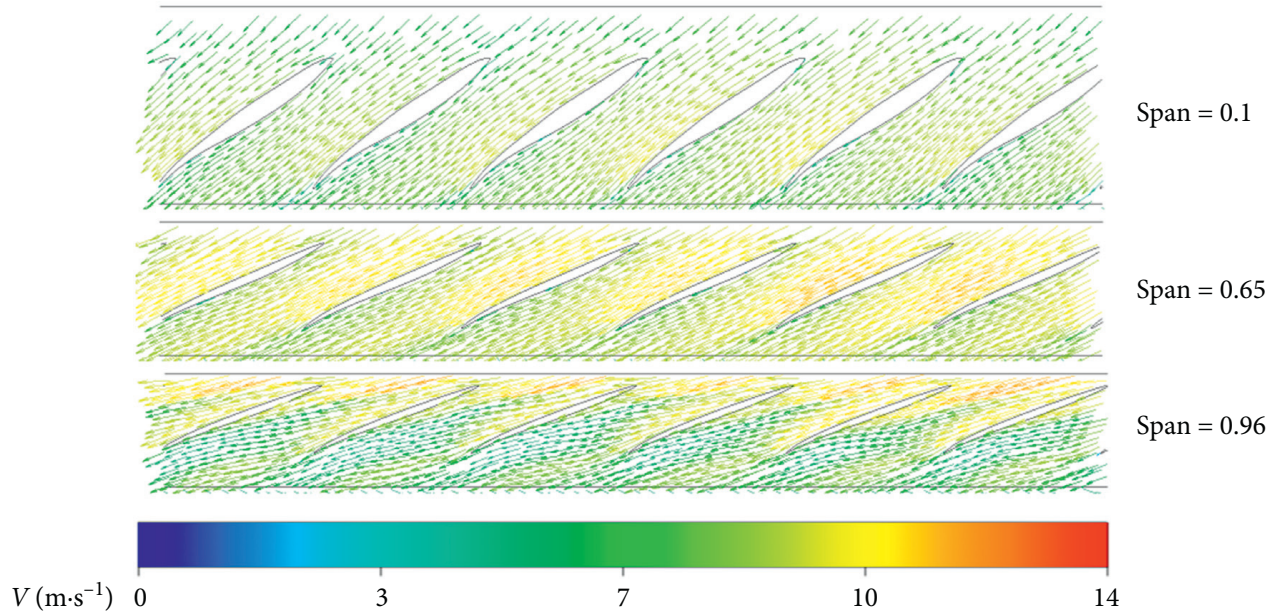

(a)
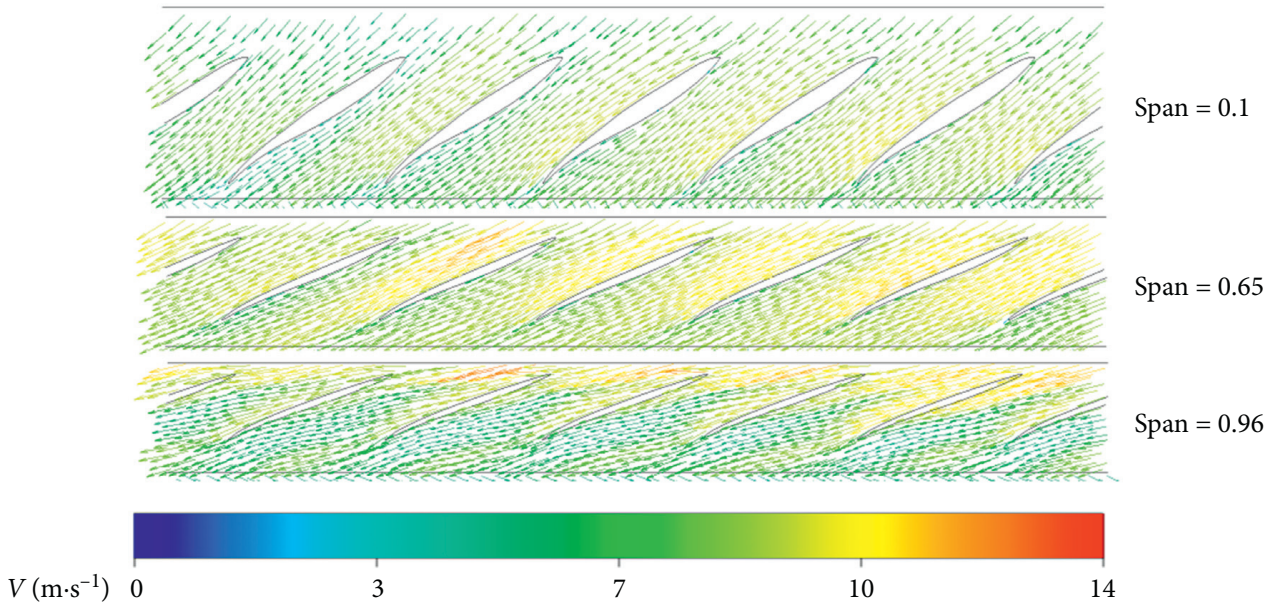

(b)

Figure 19: Velocity vector on each span of original scheme and selected scheme under $1.0 Q^{\prime}$ condition: (a) original scheme; (b) selected scheme. 
much different, and the work capacity is basically unchanged, so the head at this point is not much different.

In order to explore the flow pattern inside the impeller, the velocity vector near the hub $(\operatorname{span}=0.1)$, the center of the vortex core $(\operatorname{span}=0.65)$, and the wheel rim $(\operatorname{span}=0.96)$ on the span is taken for analysis.

Figure 17 is the velocity vector diagram of the original scheme and the selected scheme on each span under the condition of $0.5 Q^{\prime}$. Under this working condition, the flow pattern of the two schemes is smooth with $\operatorname{span}=0.1$; when span $=0.65$, the spanwise vortices are observed in the four grooves of the original scheme, and the spanwise vortices are observed in the trailing edge of the suction surface of the three grooves of the selected scheme, which is corresponding to the distribution of the blade vortices. The spanwise vortices are the embodiment of the spatial development of the vortices in the groove; when $\operatorname{span}=0.96$, both schemes have relatively serious backflow. This seriously affects the work efficiency of the blade.

Figure 18 is the velocity vector diagram on each span of the original scheme and the selected scheme under the condition of $0.67 Q^{\prime}$. In the original scheme, the flow on span $=0.1$ and $\operatorname{span}=0.65$ is relatively smooth. In the selected scheme, spanwise vortex occurs on the trailing edge of suction surface of some blades on $\operatorname{span}=0.65$. On the span $=0.96$, the velocity distribution at the inlet of the two schemes is not uniform, and the flow in the selected scheme becomes more disordered.

Figure 19 is the velocity vector diagram on each span of the original scheme and the selected scheme under the condition of 1.0 $Q^{\prime}$. The flow pattern on each span is well, and no spanwise vortex is observed. This shows that, under the selected scheme, this measure has little effect on the flow pattern in the impeller under the optimal condition.

\section{Conclusion}

It is proposed to set grooves in front of the impeller inlet to restrain the rotating stall, the orthogonal design of this measure is given, and the proper size of each design parameter is found through the orthogonal test. Through the analysis of the orthogonal test results, it can be seen that the influence of each groove parameter on the performance of the device is different. The most important influence on the performance of the device is the groove width, followed by the number of grooves and the groove depth, and the influence of the groove length is the smallest. The optimal values of each parameter are obtained, which are $7.75 \times 10^{-2} \mathrm{D}$ in length, $3.10 \times 10^{-2} \mathrm{D}$ in width, $3.10 \times 10^{-2} \mathrm{D}$ in depth, and 72 grooves. Scheme 16 is the selected scheme.

The numerical calculation of the selected groove scheme is carried out under different flow rate conditions, and the results are compared with the results of the original scheme. The results show that the scheme can improve the flow pattern in the impeller under small flow rate conditions and improve the head at the bottom of the valley. However, due to the existence of grooves, the part hydraulic loss is increased, and the overall efficiency of the selected groove scheme is lower than the original scheme. In addition, in order to analyze the suppression mechanism of the selected groove scheme, not only the total pressure coefficient on different sections is studied and compared but also the flow pattern in the groove and the reason for the disappearance of the positive slope area after the groove is added are explored. It is found that the disappearance of the positive slope area is related to the static pressure difference on the blade and mainly depends on the static pressure on the suction surface. At the same time, the flow pattern of the original scheme and the selected scheme on the spans near the hub, the center of the vortex core, and the wheel rim is also studied. It is found that the flow pattern on each span, especially the span near the vortex core center, is consistent with the head change law of the device; that is, after the flow pattern on the span near the vortex core center of the selected scheme becomes smooth, the head of the corresponding device rises; on the contrary, the head of the device decreases.

\section{Data Availability}

The data used to support the findings of this study are available from the corresponding author upon request.

\section{Conflicts of Interest}

The authors declare that there are no conflicts of interest regarding the publication of this paper.

\section{Acknowledgments}

This research was funded by the Jiangsu Province Science Foundation for Youths (Grant no. BK20170507), Natural Science Foundation of the Jiangsu Higher Education Institutions (Grant no. 17KJD580003), Jiangsu Planned Projects for Postdoctoral Research Funds (Grant no. 1701189B), Open Research Subject of Key Laboratory of Fluid and Power Machinery (Xihua University), Ministry of Education (Grant no. szjj2019-018), Science and Technology Innovation and Cultivation Fund of Yangzhou University (Grant no. 2019CXJ076), National Natural Science Foundation of China (Grant no. 51779214), Priority Academic Program Development of Jiangsu Higher Education Institutions (PAPD), Jiangsu Province 333 High Level Talents Training Project (Grant no. (2018) III-1827), and Peak Plan Six Talents in Jiangsu Province, and Key Project of Water Conservancy in Jiangsu Province (Grant no. 2018042).

\section{References}

[1] J. Wei, J. Yin, and Q. Miao, "The study on the prediction method of the ship's speed loss in the wind wave," in Proceedings of the 9th National Conference on Hydrodynamics and National Hydrodynamics, pp. 759-765, Shanghai, China, 2009.

[2] R. Motte and S. Calvert, "Operational considerations and constraints in ship-based weather routing procedures," The Journal of Navigation, vol. 41, no. 3, pp. 417-433, 1998.

[3] T. Sano, Y. Yoshida, Y. Tsujimoto, Y. Nakamura, and T. Matsushima, "Numerical study of rotating stall in a pump vaned diffuser," Journal of Fluids Engineering, vol. 124, no. 2, pp. 363-370, 2002. 
[4] L. Cheng, L. Wu, and C. Liu, "Hydraulic unstable operating region of large scale axial flow pump," Journal of Irrigation and Drainage, vol. 29, no. 2, pp. 102-104, 2010.

[5] L. Cheng and W. Qi, "Rotating stall region of water-jet pump," Transactions of Famena, vol. 38, no. 2, pp. 31-40, 2014.

[6] Z. Pan, J. Li, H. Li et al., "Overview for research on rotating stall of pump," Fluid Machinery, vol. 39, no. 2, pp. 35-39, 2011.

[7] A. Arpad, "Anslysis of separated flows in hydro machines," in Proceedings of the 6th IASME/WSEAS International Conference on fluid mechanics and aerodynamics (FMA08), pp. 105-108, Rhodes, Greece, 2008.

[8] H. Emmons, C. Pearson, and H. Grant, "Compressor surge and stall propagation," Transactions of ASME, vol. 79, pp. 455-469, 1955.

[9] H. Yang, D. Sun, F. Tang et al., "Research on the performance improvement of axial-flow pump under unstable condition using CFD," Transactions of the Chinese Society for Agricultural Machinery, vol. 43, no. 11, pp. 138-151, 2012.

[10] C. Xia, C. Cheng, C. Luo et al., "Hydraulic characteristics and measurement of rotating stall suppression in a waterjet propulsion system," Transactions of Famena, vol. 42, no. 4, pp. 85-100, 2018.

[11] H. L. Wang, B. Long, Y. Yang, Y. Xiao, and C. Wang, "Modelling the influence of inlet angle change on the performance of submersible well pumps," International Journal of Simulation Modelling, vol. 19, no. 1, pp. 100-111, 2020.

[12] X. He, Y. Zhang, C. Wang et al., "Influence of critical wall roughness on the performance of double-channel sewage pump," Energies, vol. 13, no. 2, p. 464, 2020.

[13] C. Wang, X. He, D. Zhang et al., "Numerical and experimental study of the self-priming process of a multistage self-priming centrifugal pump," International Journal of Energy Research, vol. 43, no. 9, pp. 1-19, 2019.

[14] L. Shi, J. Zhu, F. Tang, and C. Wang, "Multi-disciplinary optimization design of axial-flow pump impellers based on the approximation model," Energies, vol. 13, no. 4, p. 779, 2020.

[15] C. Wang, X. Chen, N. Qiu, Y. Zhu, and W. Shi, "Numerical and experimental study on the pressure fluctuation, vibration, and noise of multistage pump with radial diffuser," Journal of the Brazilian Society of Mechanical Sciences and Engineering, vol. 40, no. 10, p. 481, 2018.

[16] W. Jiao, L. Cheng, J. Xu, and C. Wang, "Numerical analysis of two-phase flow in the cavitation process of a waterjet propulsion pump system," Processes, vol. 7, no. 10, p. 690, 2019.

[17] H. Wang, B. Long, C. Wang, C. Han, and L. Li, "Effects of the impeller blade with a slot structure on the centrifugal pump performance," Energies, vol. 13, no. 7, p. 1628, 2020.

[18] G. Peng, X. Huang, L. Zhou, G. Zhou, and H. Zhou, "Solidliquid two-phase flow and wear analysis in a large-scale centrifugal slurry pump," Engineering Failure Analysis, vol. 114, Article ID 104602, 2020.

[19] L. Zhou, W. Wang, J. Hang, W. Shi, H. Yan, and Y. Zhu, "Numerical investigation of a high-speed electrical submersible pump with different end clearances," Water, vol. 12, no. 4, p. 1116, 2020.

[20] L. Bai, L. Zhou, X. Jiang, Q. Pang, and D. Ye, "Vibration in a multistage centrifugal pump under varied conditions," Shock and Vibration, vol. 2019, Article ID 2057031, 9 pages, 2019.

[21] J. Qian, M. Chen, X. Liu, and Z. Jin, "A numerical investigation of the flow of nanofluids through a micro tesla valve," Journal of Zhejiang University-Science A, vol. 20, no. 1, pp. 50-60, 2019.
[22] J. Qian, Z. Gao, B. Liu et al., "Parametric study on fluid dynamics of pilot-control angle globe valve," ASME Journal of Fluids Engineering, vol. 140, no. 11, p. 111103, 2018.

[23] L. Shi, W. Zhang, H. Jiao et al., "Numerical simulation and experimental study on the comparison of the hydraulic characteristics of an axial-flow pump and a full tubular pump," Renewable Energy, vol. 153, pp. 1455-1464, 2020.

[24] Y. Zhu, S. Tang, L. Quan et al., "Extraction method for signal effective component based on extreme-point symmetric mode decomposition and Kullback-Leibler divergence," Journal of the Brazilian Society of Mechanical Sciences and Engineering, vol. 41, no. 2, p. 100, 2019.

[25] H. Wang, Z. Qian, D. Zhang, T. Wang, and C. Wang, "Numerical study of the normal impinging water jet at different impinging height, based on wray-agarwal turbulence model," Energies, vol. 13, no. 7, p. 1744, 2020.

[26] J. Zhang, S. Xia, S. Ye et al., "Experimental investigation on the noise reduction of an axial piston pump using free-layer damping material treatment," Applied Acoustics, vol. 139, pp. 1-7, 2018.

[27] B. Hu, X. Li, Y. Fu et al., "Experimental investigation on the flow and flow-rotor heat transfer in a rotor-stator spinning disk reactor," Applied Thermal Engineering, vol. 162, Article ID 114316, 2019.

[28] Z. Zhang, G. Yan, G. Zhu, P. Zhao, Z. Ma, and B. Zhang, "Using microwave pretreatment to improve the high-gradient magnetic-separation desulfurization of pulverized coal before combustion," Fuel, vol. 274, Article ID 117826, 2020.

[29] K. Zhang, S. Wang, Z. He, M. Wu, and X. Cao, "Study on acrylate peelable nuclear detergent for film formation at low temperature," Applied Radiation and Isotopes, vol. 162, Article ID 109187, 2020.

[30] M. Chen, Q. Fu, X. Song et al., "Preparation of resveratrol dry suspension and its immunomodulatory and anti-inflammatory activity in mice," Pharmaceutical Biology, vol. 58, no. 1, pp. 8-15, 2020.

[31] B. Zhang, Y. Ni, J. Liu et al., "Bead-immobilized Pseudomonas stutzeri Y2 prolongs functions to degrade s-triazine herbicides in industrial wastewater and maize fields," Science of The Total Environment, vol. 731, Article ID 139183, 2020.

[32] Y. Li and C. Hu, Experiment Design and Data Processing, Chemical Industry Press Co. Ltd., Beijing, China, 2017.

[33] C. Luo, H. Liu, L. Cheng, C. Wang, W. Jiao, and D. Zhang, "Unsteady flow process in mixed waterjet propulsion pumps with nozzle based on computational fluid dynamics," Processes, vol. 7, no. 12, p. 910, 2019. 